\title{
Dissipative phenomena in extended-body interactions
}

\section{Methods: Dwarf galaxies of the Local Group and their synthetic CMDs ${ }^{\star}$}

\author{
S. Pasetto ${ }^{1,2}$, G. Bertelli ${ }^{3}$, E. K. Grebel ${ }^{1}$, C. Chiosi ${ }^{4}$, and Y. Fujita ${ }^{5}$ \\ 1 Astronomisches Rechen-Institut, Zentrum für Astronomie der Universität Heidelberg, 69120 Heidelberg, Germany \\ e-mail: sm2@mssl.ucl.ac.uk \\ 2 University College London, Department of Space \& Climate Physics, Mullard Space Science Laboratory, Holmbury St. Mary, \\ Dorking Surrey RH5 6NT, UK \\ 3 INAF - Padova Astronomical Observatory, 35122 Padova, Italy \\ ${ }^{4}$ Astronomy Department, Padova University, 35122 Padova, Italy \\ 5 Department of Earth and Space Science, Graduate School of Science, Osaka University, Toyonaka, Osaka 560-0043, Japan \\ Received 30 June 2011 / Accepted 15 February 2012
}

\section{ABSTRACT}

\begin{abstract}
Aims. Dissipative phenomena occurring during the orbital evolution of a dwarf satellite galaxy around a host galaxy may leave signatures in the star formation activity and signatures in the colour-magnitude diagram of the galaxy stellar content. Our goal is to reach a simple and qualitative description of these complicated phenomena.

Methods. We develop an analytical and numerical technique able to study the ram pressure, Kelvin-Helmholtz instability, RayleighTaylor, and tidal forces acting on the star formation processes in molecular clouds. We consider it with synthetic colour-magnitude diagram techniques.

Results. We developed a method of investigating the connections between gas consumption processes and star formation processes in the context of the two extended-body interactions, paying special attention to the dwarf galaxies dynamical regime.
\end{abstract}

Key words. instabilities - plasmas - methods: numerical - galaxies: structure - galaxies: dwarf - galaxies: stellar content

\section{Introduction}

We investigate the role of dissipative phenomena in the star formation history of a dwarf satellite galaxy orbiting a primary galaxy. Our purpose is to quantify the connections between the gas consumption processes (e.g., ram pressure, Rayleigh-Taylor and Kelvin-Helmholtz, tidal forces).

Dwarf galaxies can offer a beautiful example of interactions between a major galaxy and its satellites (e.g., Peebles 1993, 1980). While orbiting these satellites, they are affected by environmental effects that shape their evolution and may affect their star formation history (e.g., Grebel et al. 2003). Their star formation history can be inferred from their colour-magnitude diagrams (CMD), see, e.g., the recent analysis by Orban et al. (2008) of Hubble Space Telescope data for the galaxies of the Local Group (LG). Generally, environmental effects are important in groups and clusters of galaxies and are believed to contribute to the origin of the density-morphology relation (e.g., Oemler 1974; Dressler 1980; Dressler et al. 1997) or to the Butcher-Oemler effect (Butcher \& Oemler 1978; Couch et al. 1998; Goto et al. 2003). The mechanism of tidal compression exerted by the gravitational potential of a galaxy cluster is known to increase the velocity dispersion of the molecular clouds falling in disk galaxies (e.g., Valluri 1993; Byrd \& Valtonen 1990), thus inducing star formation episodes (e.g., Henriksen \& Byrd 1996; Evrard 1991). Tidal compression is also responsible for the so-called galaxy-harassment phenomenon in the case of dwarf galaxies (Moore et al. 1996, 1998, 1999) or late-type systems

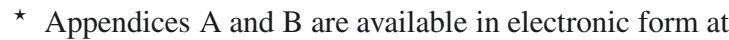
http: //www . aanda.org (e.g., Gnedin 2003). In this context, three-dimensional numerical simulations (e.g., Quilis et al. 2000) show that ram pressure can be effective in removing up to $100 \%$ of the atomic hydrogen from luminous galaxies within $10^{8} \mathrm{yr}$.

The role of the external mechanisms of gas removal in the LG dwarf galaxies has been investigated by different approaches and with conflicting results (for a review see Grebel et al. 2003) and in relation to the Milky Way (MW), we cannot neglect the dissipative role of tidal forces by the deep MW potential and its hot coronal plasma. A large amount $\left(\sim 5 \times 10^{10} M_{\odot}\right)$ of hot gas is thought to exist in an extended ( $200 \mathrm{kpc}$ ), hot diffuse halo (see e.g., Brüns et al. 2000; Sembach et al. 2003; Putman et al. 2003; Maller \& Bullock 2004; Fukugita \& Peebles 2006; Kaufmann et al. 2006; Sommer-Larsen 2006). Ram pressure can both enhance star formation by compression of the interstellar medium (ISM) (Fujita \& Nagashima 1999) and reduce it by stripping the gas from the galaxy (Gunn \& Gott 1972). Also, tidal forces can trigger episodes of star formation and its connection with the orbits has been already investigated in several papers (e.g., see recently Pasetto et al. 2011; Nichols et al. 2012).

These are the major external competitive effects that we want to investigate in the context of "dwarf galaxy-massive galaxy" interaction. In addition to this, there are also several internal mechanisms that govern the gas consumption, such as gas expulsion by star formation processes (e.g., Burkert \& Ruiz-Lapuente 1997; Ferrara \& Tolstoy 2000; Mac Low \& Ferrara 1998) giant HII regions (e.g., Davies \& Phillipps 1988; Elmegreen \& Hunter 2000). The role of the hot gaseous halo surrounding the MW has often been studied in relation with the Magellanic Stream, a long HI filament leading and trailing the Magellanic Clouds 
(see e.g., Murali 2000; Nidever et al. 2010), where the authors constrained the density of the coronal gas to be $n<10^{-5} \mathrm{~cm}^{-3}$ at the distance of $50 \mathrm{kpc}$ in order to let the Stream clouds survive up to $2.5 \mathrm{Gyr}$ or at least a minimum of $0.5 \mathrm{Gyr}$. Stanimirović et al. (2002) suggest a limit of $n \cong 3 \times 10^{-4} \mathrm{~cm}^{-3}$ at the same distance, which essentially agrees with the more recent measurements by Heitsch \& Putman (2009). Previous determinations by Kalberla \& Kerp (1998) based on the ROSAT X-ray observations agreed on the same limit for the MW halo, whereas models predicting that dwarf galaxies lose most of their gas (e.g., Gallart et al. 2001; Einasto et al. 1974) need a gas density one order of magnitude higher. On the other hand, Grcevich \& Putman (2009) argue that ram pressure is one of the primary causes of the gas deficiency of dwarf satellites within $270 \mathrm{kpc}$ around the MW and M31 (see also van den Bergh 1994; Grebel et al. 2003). Finally, we assume that the current density of the intergalactic medium beyond the LG haloes is too low for ram pressure to be effective (e.g., Quilis \& Moore 2001).

The subject of our analysis is an arbitrary idealized dwarf galaxy orbiting the MW. The differential equations involved in our study are generally coupled in a non-linear way, thus not allowing us to consider the global evolution of the processes as a linear superposition of the single effects considered alone and then summed up. Nevertheless, for the sake of clarity, it is customary to subdivide the effects involved in the evolution of a dwarf galaxy into two categories: "internal" and "external"; and then to consider their superposition. We also follow this tradition also in this paper. Among the internal effects, stellar evolution processes are the most important ones because they ultimately govern the location of the stars in a CMD. The evolution of the stars and their feedback is mainly related to their mass and chemical composition. In our approach, we carefully consider stellar feedback on the dwarf galaxy stellar populations in dependence of the mass of the stars (see Sect. 2.1). Finally, in our approximation we want to consider several processes of external nature as well, mainly tidal compression, ram pressure, and RayleighTaylor and Kelvin-Helmholtz instabilities. The reasons for focusing on these effects are the following. We are mostly interested in the effects of the orbits on the generation of observable CMD. To approach this problem we work out a spherical approximation for the shape of the idealized dwarf galaxy considered as a whole. On the one hand, this strong approximation will make us lose track of the orbital evolution of the stars inside the galaxy itself; i.e. we do not solve the equation of motion (EoM) for individual stars inside the dwarf galaxy (see Sect. 2.3), but this approach will allow us to neglect the effects of tidal stripping and tidal stirring (e.g., Mayer et al. 2001; Pasetto et al. 2003; Read et al. 2006; Klimentowski et al. 2009), giving more agility to our code (see the code performance in Appendix A) than with standard $N$-body techniques (see, e.g., Mayer 2010, for a recent review). Finally, to simplify the orbit integration further, we limit ourselves to non-penetrating encounters with the MW; i.e., we limit our analysis to the dwarf galaxy orbit pericentre, $\boldsymbol{r}_{\mathrm{p}}$, greater than $\left\|\boldsymbol{r}_{\mathrm{p}}\right\|>20 \mathrm{kpc}$ (with $\|\boldsymbol{a}\|$ standard Euclidean norm of a vector $\boldsymbol{a}$ ), thus partially neglecting the problem of the indetermination of the parameters of the MW potential, the role of the dynamical friction, and role of tidal shocks due to the passages through the MW disk (not the target of the present paper). Because we are mainly focused on the LG dwarf galaxy orbits, we do not consider galaxy harassment because it is much less efficient in low-density groups such as our LG (e.g., Moore et al. 1996).

The paper is organized as follows: in Sect. 2 we present our model for gas consumption and star formation, taking both internal and environmental effects into account that would affect the star formation history, while the dwarf galaxy is orbiting a host galaxy (the MW in this paper). In Sect. 3 we briefly summarize the key lines of the population synthesis technique to generate colour-magnitude diagrams out of which the history of star formation is inferred. In Sect. 4 the technique is applied to a model of a dwarf galaxy in which the star formation efficiency is altered by the environmental effects during the orbital motion and from which a synthetic CMD is generated and briefly analysed. A brief discussion of the results and some general conclusions are presented in Sect. 5.

Finally, we made extensive use of the Appendix to present any technical test of the code and to explain the analytical aspects of the work that can be postponed until after a first reading of the paper.

\section{A model of gas consumption and star formation in dwarf galaxies}

\subsection{Molecular clouds evolution and non gravitational heating}

To link the orbital motion of a satellite about its host galaxy to the environmental effects and the star formation efficiency in the satellite itself, we must set up a fast algorithm that allows us to explore a large space of initial conditions for the orbital motions. This immediately rules out a multi-phase, fully hydrodynamic treatment of the gas (e.g., Quilis et al. 2000; Tonnesen et al. 2007) because it would be too time consuming, in particular if a large volume of initial conditions (i.c.) of the orbital motions has to be explored. Therefore, we prefer to follow the method proposed by Fujita (1998) and Fujita \& Nagashima (1999), from whom we take the model of star formation to be integrated by extensive calculations of orbital motions and synthetic CMDs of the stellar content of the dwarf galaxy.

In brief, stars are known to form in gas clouds (either atomic or molecular) whose mass distribution is uncertain but believed to span at least a factor $\sim 10^{6}$ in mass (Elmegreen \& Efremov 1997). We group the molecular clouds according to their initial mass $M_{i} \mid M_{i} \in\left[M_{\min }, M_{\max }\right]$ and assume $M_{\min } \cong 10^{2} M_{\odot}$ and $M_{\text {max }} \cong 10^{8} M_{\odot}$. Since this mass interval is very large, we split it logarithmically and resolve the mass intervals with a ratio $\alpha$ between any two subsequent intervals; i.e., we solve the recurrence relation $\log _{10} \frac{M_{i+1}}{M_{i}}=\alpha$, with the boundary condition $M_{0}=M_{\text {min }}$ for $i=0$, as $M_{i}=10^{\alpha i} M_{\min }$ ( $\alpha$ is a rational number). The numerical convergence is confirmed for $\alpha=\frac{1}{100}$ (see Appendix A for details).

For simplicity we then define a "mass class" within the generic interval $\hat{M}_{i} \equiv M_{i+1}-M_{i}$ or its fraction over a total mass $M_{\text {tot }}$ as $\hat{f}_{i} \equiv \hat{M}_{i} / M_{\text {tot }}$. We assume that the evolution of each mass class $\hat{M}_{i}$ representative of each mass interval is governed by the fraction of gas that is ejected from the stars, say their gas ejection rate $R_{\text {star }}$ and by the amount of recycled molecular gas with recycling rate $R_{\text {mol }}$.

To determine $R_{\text {star }}$ we remember that short-lived and longlived stars contribute to $R_{\text {star }}$ in a different fashion. If $r(m)$ is the amount of mass returned by a star of mass $m, \mu(m)=\frac{r(m)}{m}$ is the fractionary return mass. Then the gas ejection rate due to stars with short lifetimes, $R_{\mathrm{star}}^{[\mathrm{S}]}$, will depend on the mass distribution of the stars, i.e. the time independent initial mass function $\iota=\iota(\mathrm{m})$ expressed as a mass fraction, and the star formation rate $\psi$ at the moment when the star is born $\psi(t-\tilde{t}(m))$ with $\tilde{t}(m)$ the lifetime of a star of mass $m$. Supposing that the typical age of a galaxy is, for instance, $t_{\mathrm{G}}=12 \mathrm{Gyr}$ and looking for mass of stars whose 
lifetime is shorter than to the age of the galaxy (say $1 / 10 t_{\mathrm{G}}$ ), $R_{\mathrm{star}}^{[\mathrm{S}]}$ is given by

$R_{\mathrm{star}}^{[\mathrm{S}]}(t)=\int_{m_{\mathrm{low}}}^{m_{\mathrm{up}}} \psi(t-\tilde{t}(m)) \mu(m) \iota(m) \mathrm{d} m$

where $m_{\text {low }}=2.3 M_{\odot}$ is the star with $\tilde{t}(m) \simeq 0.1 t_{\mathrm{G}}$ and $m_{\text {up }}$ is the most massive star we want to consider $m_{\mathrm{up}}=100 M_{\odot}$. All the less massive stars contribute to the long-lived part of $R_{\text {star }}$ indicated as $R_{\mathrm{star}}^{[\mathrm{L}]}$. The contribution of these stars can be written as $R_{\mathrm{star}}^{[\mathrm{L}]}=\psi_{0}-R_{\mathrm{star}}^{[\mathrm{S}]}(0)$, where $\psi_{0}$ is an artificially fixed star formation rate for the galaxy we are considering, and we consider the lifetime corresponding to the transition mass from long-lived to short lived stars $t_{\mathrm{tr}}=0.1 t_{\mathrm{G}}$ Gyr. Recollecting our previous terms, we get

$$
\begin{aligned}
R_{\mathrm{star}} & =R_{\mathrm{star}}^{[\mathrm{S}]}+R_{\mathrm{star}}^{[\mathrm{L}]}=\theta(t)\left(R_{\mathrm{star}}^{[\mathrm{S}]}(t)-R_{\mathrm{star}}^{[\mathrm{S}]}(0)\right)+\psi_{0} \\
& =\int_{m_{\mathrm{low}}}^{m_{\mathrm{up}}}[\psi(t-\tilde{t})-\psi(-\tilde{t})] \mu(m) \iota(m) \mathrm{d} m,
\end{aligned}
$$

where $\theta(x)$ is a step function ( $\theta=0$ for $x \leq 0, \theta=1$ otherwise), and $R_{\text {star }}^{[\mathrm{L}]}$ is indeed not time-dependent for $t>0$.

Furthermore, we define the molecular recycling rate, $R_{\text {mol }}$, as the complement of the star formation efficiency, $1-\varepsilon$ where $\varepsilon$ is a general function of molecular cloud mass $M$ and pressure $P$, i.e. $\varepsilon=\varepsilon(M, P)$. The star formation efficiency in turn can be reduced by the presence of an external gas consumption mechanism, e.g., HI phase, ram pressure, Rayleigh-Taylor instability, tidal compression, etc. (see Sect. 2.4 below for a detailed discussion). Therefore, for each mass class, $i$, we can define

$R_{\mathrm{mol}}(t)=\sum_{i}\left(1-\varepsilon\left(M_{i}, P\right)\right) \frac{\hat{M}_{i}(t)}{\tau\left(M_{i}, P\right)}$,

which depends on all the molecular clouds $i$, and where $\tau\left(M_{i}, P\right)$ in the destruction time of a molecular cloud with mass $M_{i}$ and pressure $P$ (see below for an extended discussion, and Appendix B for the analytical formulation). In principle, it is now simple to introduce a reducing factor in $R_{\text {mol }}$ into Eq. (3) in order to lower the fraction $1-\varepsilon_{\text {eff }}$ when the stronger energy injection by supernovae ( $\mathrm{SNe}$ ), photo-ionization of molecular clouds, or the effects of strong stellar winds need to be investigated. We have chosen to focus the present work mostly on external effects on the gas consumption or gas removal.

Finally, the time variation of $\hat{M}_{i}$ simply depends on the lifetime $\tau$ of the molecular cloud according to the system of integrodifferential equations ${ }^{1}$ :

$$
\frac{\mathrm{d} \hat{M}_{i}}{\mathrm{~d} t}=\theta\left(\hat{M}_{i}\right)\left[\hat{f}_{i}\left(R_{\mathrm{star}}+R_{\mathrm{mol}}\right)-\frac{\hat{M}_{i}}{\tau}\right],
$$

which relates the rate of change of each mass class $\frac{\mathrm{d} \hat{M}_{i}}{\mathrm{~d} t}$ to all the other molecular classes though the gas ejection rate of the stars $R_{\text {star }}$, and the recycle rate of molecular clouds $R_{\text {mol }}$ (where we again use the step function defined after Eq. (2) to take into account that the mass is a positive definite quantity).

Once the system of Eqs. (4) is considered together with Eqs. (1)-(3), we can estimate the star formation rate (SFR) as

$\psi(t)=\sum_{i} \varepsilon\left(M_{i}, P\right) \frac{\hat{M}_{i}(t)}{\tau\left(M_{i}, P\right)}$,

\footnotetext{
1 To reduce the degrees of complexity of the equations, we do not consider cosmological effects or the explicit dependence of the equations on the redshift.
}

which relates the evolution of the $i^{\text {th }}$ molecular cloud to all the other $N-1$ molecular clouds as seen in Eq. (1) and represents the target of our computation.

In the previous system of equations the only functions that need to be adopted from the literature are the star formation efficiency of a molecular cloud $\varepsilon\left(M_{i}, P\right)$ and the destruction time $\tau\left(M_{i}, P\right)$ of a molecular cloud with mass $M_{i}$ and pressure $P$. Then, once these functions are assumed, the system can be integrated once $\psi_{0}$ is assumed on the basis of the system we want to analyse (e.g., globular clusters, dwarf galaxies, spiral galaxies, etc.).

Star formation most likely responds to the pressure variation in the local interstellar medium, and in our case it is worth recalling that the pressure $P=P(\boldsymbol{x}, t)$ depends on time and relative position with respect to the host galaxy (with the barycentre centred on the origin $O$ of the inertial reference system $S_{0}$ with vector radius $x$ ) and the environment. The relation between star formation efficiency, $\varepsilon$, mass $M$ and pressure $P$ of molecular clouds has been studied e.g., in Elmegreen \& Efremov (1997) and Krumholz \& McKee (2005) showing how a pressure increase causes an increment of $\varepsilon$ and a subsequent decrease in the lifetime of a cloud $\tau$. These relations are independent of the physical origin of the pressure and can be safely applied to our treatment of the star formation history (SFH) during the orbital motion of a dwarf galaxy. We simply assume these relations from the literature. A plot of their values can be seen in Fig. 1 for the range of values adopted in the case of the dwarf orbiting the MW. We refer the interested reader to further details and their analytical definition in Appendix B.

Finally, we mention that the dynamical response to gas removal by $\mathrm{SNe}$ explosions in dwarf galaxies has been repeatedly invoked as a mechanism to suppress the star formation in these objects (e.g., Somerville \& Primack 1999; Cole et al. 2000; De Lucia et al. 2004; Nagashima \& Yoshii 2004; Kang et al. 2005). These results nevertheless contrast with observational properties, as pointed out in Steinmetz \& Navarro (1999), Navarro \& Steinmetz (2000a), Navarro \& Steinmetz (2000b), Portinari \& Sommer-Larsen (2007) suggesting that numerical recipes can be improved, as recently shown by Pasetto et al. (2010) using prescriptions based on chemo-dynamical studies. These authors made it clear that gas survival in spherical dwarf galaxies is perfectly compatible with the expected $\mathrm{SNe}$ rate over the whole mass range of interest for dwarf galaxies (see their Table 1). This reasonably stems from the reduced SNe feedback expected in the local environment (Cioffi \& Shull 1991) different from dwarf to spiral galaxies (Bradamante et al. 1998), and it explains the co-existence of old stellar populations and a reservoir of neutral gas in a natural way (e.g., Phillipps et al. 1990; Pasetto et al. 2010).

\subsection{Galaxy model and plasma distribution}

The most important contributions to understanding the plasma corona of the MW come from particle physics (e.g., Murali 2000) and cosmology (e.g., Fukugita \& Peebles 2006). Nevertheless, the literature is rich in attempts to model the distributions of the hot gas density (which because of the high degree of ionization refers uniquely to electron density) in different environments e.g. in the LG (Rasmussen \& Pedersen 2001; Binney \& Evans 2001; Maloney \& Bland-Hawthorn 1999) by considering different constraints, in clusters of galaxies by fitting the X-ray observations with $\beta$-models (e.g., Cavaliere \& Fusco-Femiano 1976) and by fitting the MW hot coronal gas (e.g., Moore \& Davis 1994; Fukugita \& Peebles 2006; Richter et al. 2008; Savage et al. 2003; Tumlinson \& Fang 2005). In a 


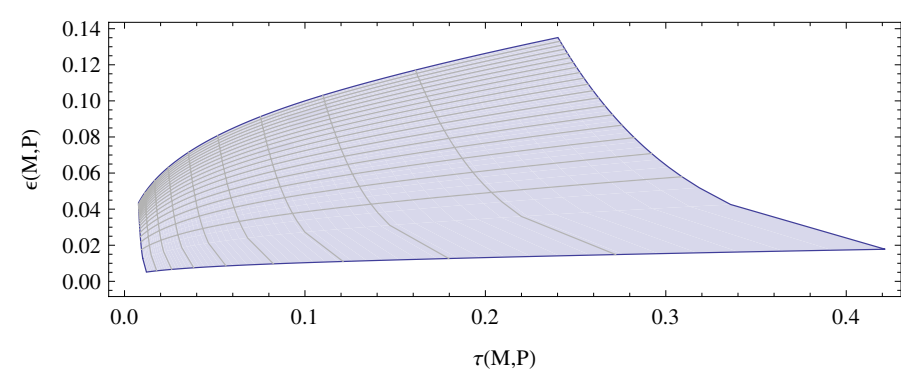

Fig. 1. Star formation efficiency vs. lifetime relation. The surface is an interpolation with 20 meshes of constant pressure run horizontally and linearly for $P \in[0.001,1] P_{\odot}$ from top to bottom. Ten mesh lines run vertically for constant mass, logarithmically for $\hat{M}_{i} \in\left[10^{2}, 10^{6}\right] M_{\odot}$ from left to right.

hierarchical galaxy formation scenario, after the initial collapse, the continuing accretion of gas-rich fragments can produce a diffuse hot gas halo that surrounds the galaxies and fills the dark matter potential (White \& Rees 1978; White \& Frenk 1991), but not necessarily with the same radial profiles (Fukugita \& Peebles 2006). This scenario also agrees with the case of clusters of galaxies where the X-ray distribution of the intra-cluster medium is significantly flatter than the dark matter distribution, in particular for low-temperature clusters (e.g., Arnaud \& Evrard 1999; Ettori \& Fabian 1999; Mohr et al. 1999), due to non-gravitational heating.

In our modelling, we assume that the density distribution of this gas settles down in hydrostatic equilibrium from the effect of the gravity on the dark matter component of the host galaxy (the MW in our case) with a temperature around $10^{6 \div 7} \mathrm{~K}$ (or $\sim 0.086 \div 0.86 \mathrm{keV}$ as is common in plasma physics). The ion component provides the density, and it is self-consistently added to the galactic potential described by Pasetto et al. (2011), to which the reader should refer for a the detailed description. Following Fukugita \& Peebles (2006) (see also, Anderson \& Bregman 2010), we adopt $Y=0.25$ for the primordial abundance of helium, with a sound speed $v_{\mathrm{s}}=\sqrt{\gamma Z k_{\mathrm{B}} T_{\mathrm{e}} / m_{i}} \cong$ $600 \mathrm{~km} \mathrm{~s}^{-1}$ (with $T_{\mathrm{e}}$ as the temperature in $\mathrm{eV}, m_{i}$ as the ion mass, $Z$ as the charge state, $k_{\mathrm{B}}$ as the Boltzmann constant and $\gamma$ as the adiabatic index (see also Kippenhahn \& Weigert 1994). The resulting model for the electron density is shown in Fig. 2. Other approaches based on polytropic equations of state cannot add any significant insight into the problem due to the lack of observational constraints on the plasma temperature's spatial gradient or rotation in the range of distances we are interested in sampling.

\subsection{Tidal approximation for a pressure equation in non inertial reference systems.}

We limit ourselves to the simple case of a spherical dwarf galaxy whose size is given by a typical scale radius, $r_{\mathrm{s}}$, e.g., the tidal radius or the effective radius, or any other radial scale length that might be obtained from observational data. If the dwarf galaxy is not a sphere, then the direction of motion must also be specified in its orbit around a massive companion in relation to the shape of the dwarf galaxy, so more sophisticated formulations are required.

We want to develop a formalism for the pressure acting on a dwarf galaxy while orbiting through the hot coronal gas of the MW (Sect. 2.2). Quantifying the pressure is necessary in order

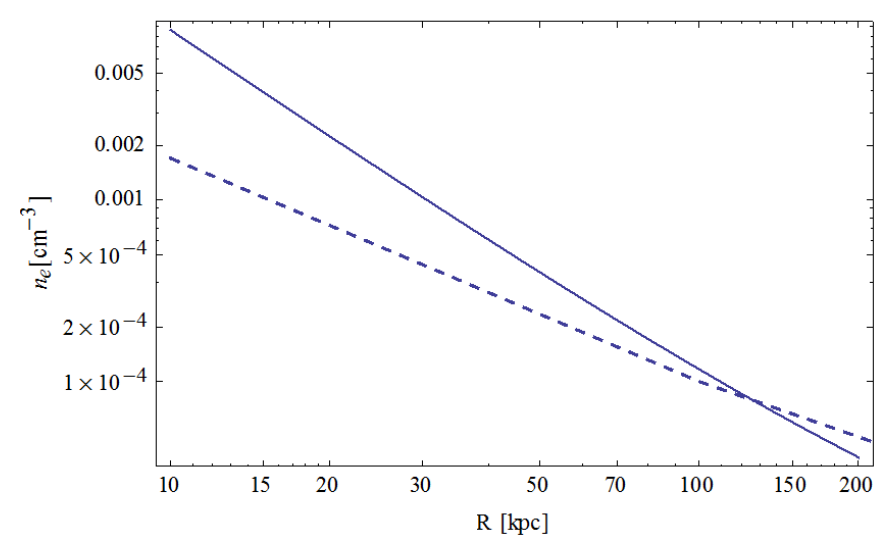

Fig. 2. Electron number density for our coronal model. For comparison the dashed line shows the electron number density model taken from the literature (Fukugita \& Peebles 2006, their "model 2").

to investigate the effects on the star formation rate as explained in Sect. 2.1.

The pressure can be obtained from the Navier-Stokes equations in a potential-flow approximation once these equation are written in a non-inertial reference system, $S_{1}$, comoving with the dwarf galaxy. This approach is based on the classical assumption that the interstellar medium (ISM) of a dwarf galaxy is much denser than the hot intergalactic medium (HIGM) of the MW. If we consider that the HIGM has a greater temperature than 10/100 times the dwarf galaxy ISM temperature (see Sect. 2.2) then we can neglect the effect of the dwarf potential on the HIGM. Moreover, the ISM will be considerably denser than the HIGM so that, to a first approximation, the flow of the hot gas is like that around a rigid body (e.g., Nulsen 1982, and references therein). In this case, we can proceed by adopting the standard formulation for the potential flow

$\varphi_{v_{0}}=-\langle\boldsymbol{v}, \boldsymbol{\xi}\rangle\left(1+\frac{1}{2} \frac{r_{\mathrm{s}}^{3}}{\|\boldsymbol{\xi}\|^{3}}\right)$

(e.g., Chorin \& Marsden 1979; Batchelor 2000). Here $\langle\boldsymbol{a}, \boldsymbol{b}\rangle$ is the inner product between two arbitrary vectors $\boldsymbol{a}$ and $\boldsymbol{b}$, the subscript $\boldsymbol{v}_{0}$ refers to the fluid velocity of the potential flow given by $\boldsymbol{v}_{0}=\nabla \varphi_{v_{0}}, \boldsymbol{v}$ is the dwarf galaxy velocity in an inertial reference system $S_{0}$ centred on the $\mathrm{MW}$, and $\boldsymbol{\xi}$ the vector radius in the non-inertial reference system $S_{1}$ whose origin is in the barycentre of the dwarf galaxy $O^{\prime}$. Hereafter the prime-notation refers to the non-inertial reference system $S_{1}$. The integration of the Euler equation is a standard procedure (e.g., Landau \& Lifshitz 1959; Batchelor 2000) that leads to the classical Bernoulli equation that we would need to solve for the pressure $P$, but instead, we write it in a non-inertial reference system by adding the extra term $\langle\boldsymbol{A}, \boldsymbol{\xi}\rangle$ that takes the non-inertial character of the reference system $S_{1}$ into account (where $\boldsymbol{A}$ is the acceleration of a fluid element due to the motion of the galaxy). We get

$\frac{\partial \varphi_{\boldsymbol{v}_{0}^{\prime}}^{\prime}}{\partial t}+\frac{P}{\rho}+\frac{\left\|\boldsymbol{v}_{0}^{\prime}\right\|^{2}}{2}=f(t)-\Phi_{\mathrm{g}}-\langle\boldsymbol{A}, \boldsymbol{\xi}\rangle$.

In this equation, $\rho$ is the coronal gas density distribution and $\Phi_{\mathrm{g}}$ the gravitational potential acting on the fluid elements. We use a prime to indicate that the potential flow now refers to a noninertial reference frame $S_{1}$, as well as its velocity $\boldsymbol{v}_{0}$. This equation closely resembles the classical Bernoulli equation except for the non-inertial term (see Sect. 3.1 of Pasetto \& Chiosi 2009, for 
a more extended discussion). Finally, we fix the arbitrary $f(t)$ (constant over all the streamlines for inviscid irrotational fluids) by imposing the boundary condition that the hot gas corona is in hydrostatic equilibrium. We easily obtain

$f(t)=\frac{\|\boldsymbol{v}\|^{2}}{2}$.

The last approximation we want to introduce before solving Eq. (7) for pressure $P$ is the tidal approximation. Because we are interested in the pressure acting in the regions close to the dwarf galaxy, we perform a Taylor expansion of the total gravitational potential $\Phi_{\mathrm{g}}(\boldsymbol{x})$ acting on the generic element of the fluid located at $\boldsymbol{x}$ close to the barycentre (located at $\boldsymbol{R}_{0}$ ) of the dwarf galaxy,

$\Phi_{\mathrm{g}}(\boldsymbol{x}) \simeq \Phi_{\mathrm{g}}\left(\boldsymbol{R}_{0}\right)+\nabla \Phi_{\mathrm{g}}\left(\boldsymbol{R}_{0}\right)\left(\boldsymbol{x}-\boldsymbol{R}_{0}\right)+\ldots$,

so that $\boldsymbol{x}-\boldsymbol{R}_{0}=\boldsymbol{O} \boldsymbol{\xi}$ with $\boldsymbol{O} \in S O(3)$ is the generic rotation matrix between the orthonormal basis of the inertial reference system $S_{0}$ and $S_{1}$ with transpose $\boldsymbol{O}^{\mathrm{T}}$, and the approximation holds for $\boldsymbol{\xi} \ll \boldsymbol{R}_{0}$ and $\boldsymbol{\xi} \ll \boldsymbol{x}$. Considering Eq. (9) and that $\boldsymbol{A}=-\nabla \Phi_{\mathrm{g}}$, we easily obtain from Eq. (7) the required equation for the pressure in the tidal approximation,

$P=\rho\left[\frac{v^{2}}{8}\left(4-9 \sin ^{2} \vartheta\right)-\Phi_{\mathrm{g}}\left(\boldsymbol{R}_{0}\right)+r_{\mathrm{s}}^{2}\left\langle\boldsymbol{O}^{\mathrm{T}} \boldsymbol{T} \boldsymbol{O} \hat{\boldsymbol{n}}, \hat{\boldsymbol{n}}\right\rangle\right]$,

where $v^{2}=\|\boldsymbol{v}\|^{2}$ is the square of the norm of the velocity vector of the dwarf galaxy, $\vartheta$ the angle between the direction of the velocity vector and the normal $\hat{\boldsymbol{n}}$ (e.g., Landau \& Lifshitz 1959), and the equation is evaluated at the scale radius: $\boldsymbol{\xi}=r_{\mathrm{s}} \hat{\boldsymbol{n}}$ with $\hat{\boldsymbol{n}}=\frac{\boldsymbol{\xi}}{\|\xi\|}$. Finally, we have introduced the tidal tensor $\boldsymbol{T}=-\boldsymbol{H}\left(\Phi_{\mathrm{g}}\left(\boldsymbol{R}_{0}\right)\right)$, defined as the negative to the Hessian matrix $\boldsymbol{H}$ in the Taylor expansion of Eq. (9) stopped at the second order in the potential and evaluated at the dwarf galaxy barycentre position.

Equation (7) is completely determined once the orientation of the reference system $S_{1}$ with respect to $S_{0}$ is defined, i.e. when we define the rotation matrix $\boldsymbol{O}$. We use the Cayley-Klein parameters (e.g., Goldstein et al. 2002) to orient $S_{1}$ with the FrenetSerret reference frame $\left\{\hat{\boldsymbol{e}}_{\mathrm{T}}, \hat{\boldsymbol{e}}_{\mathrm{N}}, \hat{\boldsymbol{e}}_{\mathrm{B}}\right\}$ once the orbit is numerically integrated where the tangent vector $\hat{\boldsymbol{e}}_{\mathrm{T}}$, the normal $\hat{\boldsymbol{e}}_{\mathrm{N}}$, and the binormal $\hat{\boldsymbol{e}}_{\mathrm{B}}$ are its instantaneous associated unitary vectors. From the orbit we can infer the kinetic pressure $P=P(\boldsymbol{x}, t)$, where $\boldsymbol{x}=\boldsymbol{x}(t)$ is the solution of the EoM in the inertial reference system $S_{0}$ centred on the host galaxy defined by the MW gravitational potential $\Phi_{\mathrm{g}}$ as in Pasetto et al. (2011) at $t_{\mathrm{lb}}=0$, i.e. the present-day potential (with $t_{\mathrm{lb}}$ look-back time). For simplicity in the following, we neglect the time dependence of the gravitational potential.

We highlight a few characteristics of Eq. (10):

- It contains an explicit dependence on the size of the system $r_{\mathrm{s}}$. This parameter can be easily changed from the tidal radius to the half-mass radius, or can be related to the light profile depending on the specific applications.

- It shows a dependence on the direction $\hat{\boldsymbol{n}}$ along which we want to estimate the pressure.

- It reduces to the standard results in the special case of the absence of a gravitational field, in the case of an inertial system of reference and for circular orbits.

In Fig. 3 we show the pressure in the reference system $S_{1}$ for a dwarf galaxy orbiting with an eccentricity $e=0.5$ and pericentre $r_{\mathrm{p}}=200 \mathrm{kpc}$ in the MW external potential. This model
Fig. 3. Plot of the pressure acting on the "surface" of a dwarf galaxy (blue sphere) with orbit E050 at the look-back time $t_{\mathrm{lb}}=9 \mathrm{Gyr}$ (see the text for further details and Fig. 5). Orange pistons represent the pressure intensity on the dwarf, where the higher the piston, the higher the pressure. Yellow shadows span $\vartheta \in[-40,40]$ deg in steps of 10 deg and are the angular validity from standard potential flow approximation.

of the galaxy is later referred to as model E050 (see further details in Fig. 5). Along each direction perpendicular to an ideal surface enclosing the ISM of the dwarf galaxy (indicated by the blue colour), a cylinder or piston is plotted. Its height is proportional to the intensity of the pressure normalized to the value at the stagnation point (the ideal point where the flow velocity vanishes). Owing to the complexity of the terms in Eq. (10), the behaviour of the cylinder is clearly not linear.

Finally, we can refine our model further in two way:

- By considering the variability of the scale radius that can occur during the evolution of the dwarf galaxy in its tidal interacting with the host system. In this case, the linearity of the Laplace equation, means we can simply add the potential flow of a reducing size dwarf

$\varphi_{v_{0}}=-\frac{\dot{r}_{\mathrm{s}} r_{\mathrm{s}}^{2}}{\|\xi\|}$

to the previous potential flow of Eq. (6). In this case to deduce the pressure term is more tedious but straightforward and we omit it.

- By considering that the orbits of dwarf galaxies are different in nature according to whether their velocity is subsonic or transonic/supersonic. When the galaxy velocity becomes comparable to or exceeds that of the sound in the inter-galactic medium, effects of the compressibility of the fluid become important, and shock waves can occur within the galaxy or backward throughout the ISM (e.g., Landau \& Lifshitz 1959). In the literature these concepts find application in problems related to the free fall of galaxies in rich clusters of galaxies (e.g., Henriksen \& Byrd 1996, their Eq. 6). Nevertheless, we do not encounter this situation in the dwarf galaxies orbiting through the hot MW coronal gas that we are going to analyse since the velocity and density involved are far from the supersonic regime. We refer the interested reader to Appendix A.3 for the test of the pressure equation and its relation to the standard supersonic formalism. 
In the panels of Figs. 4-6 we plot three representative orbits and the relative pressure computed with Eq. (10) along the direction of motion $\hat{\boldsymbol{e}}_{\mathrm{T}}$. The pressure is plotted for the unitary scale parameter $r_{\mathrm{s}}=1$, normalized pressure, and time in Gyr. As arbitrary normalization value for the pressure, we have chosen the standard literature value for pressure from the random motion of molecular clouds in the solar neighbourhood, $P_{\odot}=3 \times 10^{4} k_{\mathrm{B}} \mathrm{cm}^{-3} \mathrm{~K}$ with $k_{\mathrm{B}}$ the Boltzmann constant (e.g., Elmegreen \& Efremov 1997; Fujita 1998, and Appendix B). As expected, the maximum pressure experienced by the dwarf is at the point of closest passage in the orbit, mostly as a result of the higher velocity of the dwarf. The orbit selection is based on the eccentricity. We assume a starting distance of $200 \mathrm{kpc}$ in the MW potential described by Pasetto et al. (2011), then search for initial conditions of the orbit in the MW plane supposed to be at rest in $S_{0}$ starting from $\boldsymbol{w}_{0}=\left\{\boldsymbol{x}_{0}, \boldsymbol{v}_{0}\right\} \mid e=\{0.25,0.50,0.75\}$ with initial conditions $\boldsymbol{x}_{0}=\{0,200,0\} \mathrm{kpc}, e \equiv r_{\mathrm{a}}-r_{\mathrm{p}} / r_{\mathrm{a}}+r_{\mathrm{p}}$ where $r_{\mathrm{a}}, r_{\mathrm{p}}$ are the apocentre and pericentre of the orbits, respectively. We refer to the three orbits evolved in the MW model introduced in the previous section by their eccentricity E025, E050, E075.

\subsection{External gas consumption effect: ram pressure stripping, Kelvin-Helmholtz and Rayleigh-Taylor instability}

Given the prescription for the pressure along an assigned orbit, we can reconstruct the SFH of the galaxy by resolving the system of Eqs. (4). However, we also need to consider the effects of external instabilities that can affect the gas consumption processes and, as a consequence, the SFH of a dwarf galaxy as well. We consider two distinct types of stripping processes: (i) instantaneous stripping due to ram pressure and Rayleigh-Taylor instabilities and (ii) continuous stripping due to the Kelvin-Helmholtz instability.

The approach adopted to deal with the kinds of stripping is different. For the ram pressure and Rayleigh-Taylor instabilities, we extend the classical recipe by making use of the formalism developed in the previous section. It has the advantage of taking the galaxy size and the direction relative to the motion into account analytically. In this way, we generalize the literature results, see e.g., the classical study by Gunn \& Gott (1972) and the recent works by Roediger \& Hensler (2005) for disk galaxies or those by Mori \& Burkert (2000) on the thermal pressure in the galactic centre.

Stripping of gas by ram pressure occurs when the pressure times the unit area, i.e. $-P_{\text {gas }} \hat{n} \mathrm{~d} A$, is greater than the restoring gravitational force for a unit mass of gas (HI or molecular, see e.g., Krumholz et al. 2008, 2009; McKee \& Krumholz 2010) of the dwarf galaxy clouds (with $\Phi_{\mathrm{dg}}, M_{\mathrm{dg}}$, and $\rho_{\mathrm{dc}}$ the potential, mass, and density respectively in $S_{1}$ of the dwarf galaxy):

$$
\begin{aligned}
& \mathbf{g}(r)=-\frac{\mathrm{d} \Phi_{\mathrm{dg}}}{\mathrm{d} \xi} \hat{\boldsymbol{n}}=-\frac{G M_{\mathrm{dg}}(\xi, t)}{\xi^{2}} \hat{\boldsymbol{n}} \\
& M_{\mathrm{dg}}(\xi, t)=4 \pi \int_{0}^{r_{\mathrm{s}}} \rho_{\mathrm{dg}}(\xi, t) \xi^{2} \mathrm{~d} \xi .
\end{aligned}
$$

For simplicity, the time dependence is only in the total mass derived from Eqs. (4), whereas the scale of the structural parameters remains unchanged.

This formalism can be easily applied to studying the Rayleigh-Taylor instability that in order to occur requires

$$
\frac{G M_{\mathrm{dc}}(\xi, t)}{\xi^{2}} M_{\mathrm{gas}}(\xi, t)<\frac{1}{2} c_{\mathrm{d}} P,
$$

where the drag coefficient, $c_{\mathrm{d}}$, is taken to be equal to one for simplicity (e.g., Roediger \& Hensler 2008). It is also interesting to note that the formulation adopted in Eq. (10) is "local", in the sense that it contains information regarding the direction $\hat{\boldsymbol{n}}$ of the point where the condition Eq. (13) holds. In this way we can take the direction of observation $\hat{\boldsymbol{n}}$ into account. We see here in an analytical way which dwarf galaxy parameters are relevant for to Rayleigh-Taylor instability. This effect has a dependence on the velocity of the dwarf $v$, the scale length $r_{\mathrm{s}}$ and the direction of the motion through the function $\sin \vartheta$. As soon as we look away from the direction of the velocity vector (i.e. $\vartheta$ increases), there are zones of the dwarf galaxy that are more stable against this type of instability i.e., the pressure in Eq. (13) decreases. At the same way we see that the more massive the galaxy is, the less efficient is Rayleigh-Taylor stripping.

Finally, the gas surviving the instantaneous ram pressure stripping can be later removed by the Kelvin-Helmholtz instabilities occurring at the interfaces between the ISM in the dwarf galaxy and the HIGM, even if their effects are partially suppressed by gravity. Since we are not interested in wakes we can safely neglect the ISM viscosity (Roediger \& Brüggen 2008) and consider this stripping process as continuous and mostly affecting the gas in the flow past the dwarf galaxy (Nulsen 1982). This effect is expected to decrease the total mass of the dwarf galaxy in the Eqs. (4) according to

$$
\left.\frac{\mathrm{d} M}{\mathrm{~d} t}\right|_{\mathrm{KH}}=\pi r_{\mathrm{s}}^{2} \rho v,
$$

with a natural time-scale $\tau_{\mathrm{KH}}=M_{\mathrm{gas}} /\left.\frac{\mathrm{d} M}{\mathrm{~d} t}\right|_{\mathrm{KH}}$ and an instability condition that comes from the classical perturbative approach (e.g., Chandrasekhar 1961)

$k>\frac{g}{v^{2}}\left(\frac{1}{\rho_{\mathrm{HIGM} / \rho_{\mathrm{ISM}}}}-\rho_{\mathrm{HIGM} / \rho_{\mathrm{ISM}}}\right)$

with $k$ wavenumber of the dominant wavelength gas ablation for Kelvin-Helmholtz instability assumed to be of the order of the scale parameter $r_{\mathrm{s}}$ (e.g., Murray et al. 1993) and $g=\|\mathbf{g}\|$ from Eq. (12).

\section{Synthetic colour-magnitude diagrams}

In order to explore the connection between the SFH of dwarf galaxies and their physical causes which may be either internal (i.e. star formation itself and feedback) or external (environment processes: i.e. orbits, tidal field and gas dissipative phenomena), we have to deal with the constraints imposed by the observations. The most direct detection of the SFH is based on the study of CMDs with the aid of the synthetic CMDs technique. In brief, given a star formation rate (SFR), an initial mass function (IMF), a binary fraction and a chemical enrichment law (expressed by the helium to heavy element ratio $\Delta Y / \Delta Z$ ), one can generate synthetic CMDs in any photometric system to be compared with their observational counterparts. To this aim, we make use of the Padova synthetic CMD generator (e.g., Ng et al. 2002). Originally this method was developed for testing stellar evolution models of stars of different mass (e.g., Bertelli et al. 1985) or stellar clusters (Chiosi et al. 1989; Bertelli et al. 1995). We use here the most recent version of this tool, which utilizes of the library of stellar models calculated by Bertelli et al. (2009). We refer to the rich literature developed by the Padova's astrophysics group for further details on YZVAR, the implementation techniques and the stellar models in use. 




Age
0
-1
-2
-3
-4
-5
-6
-7
-8
-9
-10

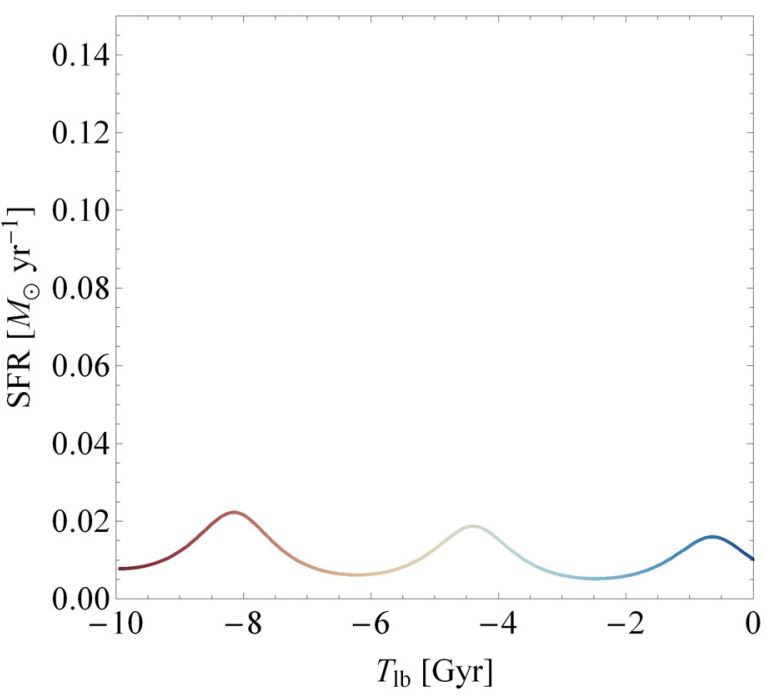



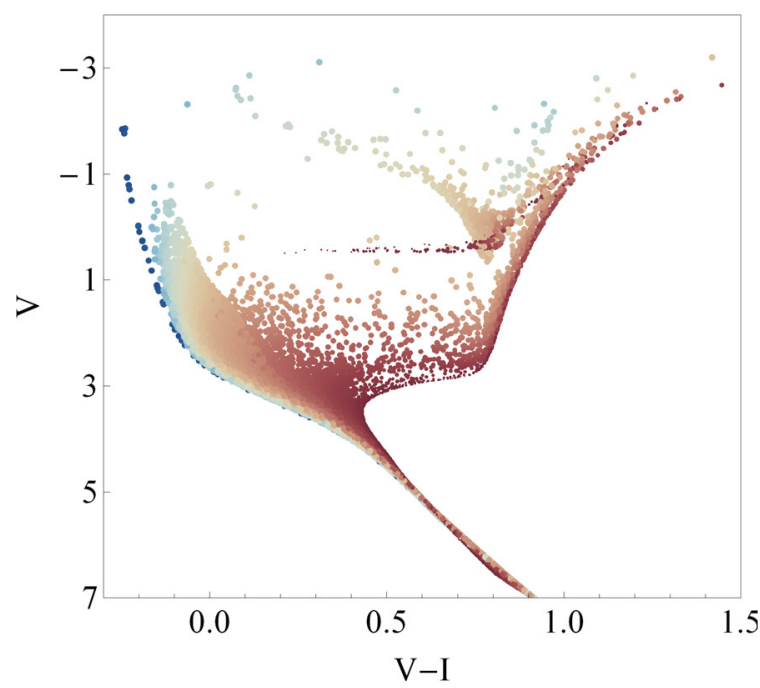

Fig. 4. The path (upper left panel), the resulting pressure profile (upper right panel) normalized to $P_{\odot}$ defined in the text, the star formation rate (lower left panel) and the resulting CMD (lower right panel) for a test satellite orbit with eccentricity $e=25$ and baryonic mass $M_{\mathrm{b}} \cong 10^{7} M_{\odot}$. The colour bar is plotted only in the upper left panel, red for the oldest stars at the beginning of the orbital path $t=0$, blue for the youngest stars born after $10 \mathrm{Gyr}$ of orbital evolution. $t_{\mathrm{lb}}$ is the look-back time in Gyr. In the CMD the size of the dots is proportional to the metallicity $\mathrm{Z}$ of the stars as obtained the Padova CMD simulator YZVAR fed with the stellar models of Bertelli et al. (2009) (metal rich stars are indicated by large dots).

In the lower right panels of Figs. 4-6 we show synthetic CMDs in the $V-I$ pass-bands without correction for the distance modulus. To study the effects of different orbits on the SFH and CMD in turn, we adopt always the same model for the dwarf galaxy, evolved along orbits with different eccentricities and leave all other parameters unchanged.

By comparing the CMD for the models (E025, E050, and E075), it is evident that SFH and associated CMDs depend on the orbit eccentricity. The CMD of the E025 model shows continuity between the episodes of star formation, which correspond to continuous gas consumption. This correlates with the orbital energy of the dwarf galaxy in two different ways. On one hand, we have a dependence on the location of the dwarf, i.e. on the configuration space. The pericentre of the dwarf galaxy is not as close to the host galaxy as for the other simulations with higher eccentricity (cf. Figs. 5 and 6). According to Eq. (10) this reduces the influence of the external tidal field and coronal gas via the potential $\Phi$ and density $\rho$. On the other hand, there is a quadratic dependence on the velocity $\propto v^{2}$, with a maximum at the pericentre passage that enhances the ram pressure stripping. Therefore, the MW dwarf galaxies with lower eccentricity (say up to $e=0.25$ ) are expected not to show clear signatures in the gas consumption of effects of a dynamical nature in their CMDs.

Different results are obtained for higher eccentricities, cf. the CMD of Fig. 6. In this case, we see different subgiant branches as signatures of an irregular consumption of the gas. Up to three to four episodes of SF are evident. The old metal-poor stars (cyan colour code and small symbols) are clearly separated from the young and metal-rich ones (red code and larger symbols). Despite the overlap of the main sequences (MSs) that should be analysed with finer resolution to resolve the sub-components, the three to four sub-giant branches (SGBs) clearly indicate three to four episodes. The lowest and faintest SGB corresponds to stars of about $0.8 M_{\odot}$ and $9 \mathrm{Gyr}$ among which older and lighter stars are also present. This is followed by several episodes, up to ages of about $\sim 3.5 / 4.0 \mathrm{Gyr}$ and masses of about $m \simeq 1.0 M_{\odot}$. Episodes younger than this are difficult to disentangle. It is beyond the aims of this study to analyse the CMD in a very detailed 




Age
0
-1
-2
-3
-4
-5
-6
-7
-8
-9
-10

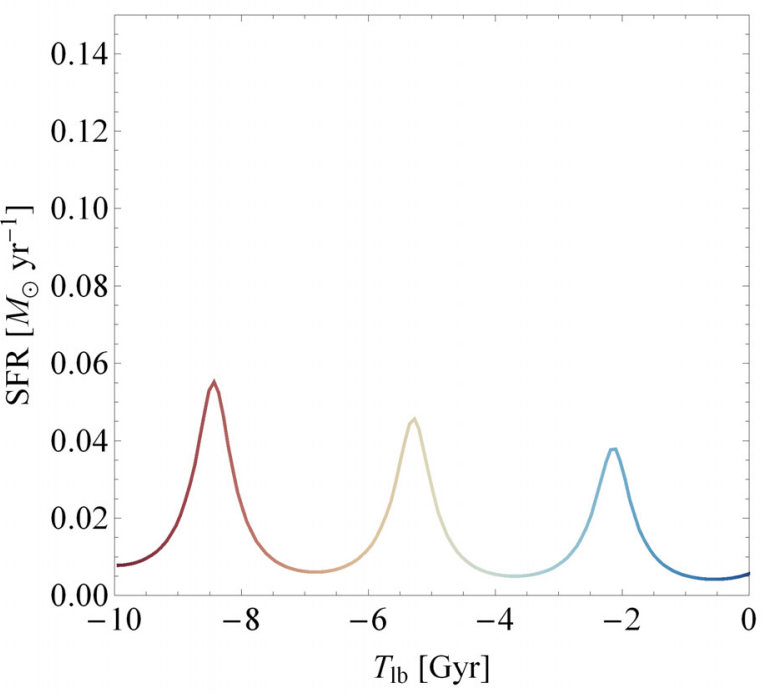

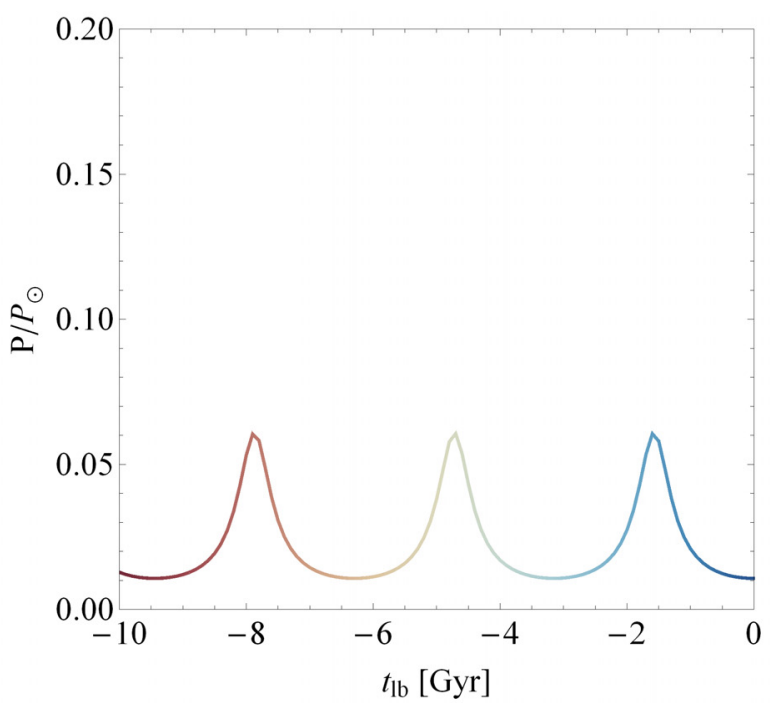



Fig. 5. As in Fig. 4 but for an orbital eccentricity $e=0.5$ of the satellites. The pressure panel refers to the stagnation point and $t_{\mathrm{lb}}$ is the look-back time in Gyr. For $t_{\mathrm{lb}}=9 \mathrm{Gyr}$ the surface pressure is represented in Fig. 3.

fashion. Our main purpose here is to show that dynamical effects can in principle leave traces in the CMDs of the stellar content of a dwarf galaxy; see Crnojević et al. (2011) and Sect. 5 below.

\section{Macrophysics vs. microphysics. An application to the Local Group dwarf galaxy Carina}

In the literature there are different approaches to studying the dissipative phenomena in the astrophysics context. The motivation of these studies is to explain observational evidence with perturbative approaches to the Navier-Stokes equations (for a review see, e.g., Chandrasekhar 1961). In relation to the astrophysics of galaxies, these works have been developed in connection with integrated properties (e.g., total mass, central velocity dispersion, etc.) and star formation processes (e.g., Livio et al. 1980; Nulsen 1982). Recently, thanks to the continuous development of the numerical techniques, we see strong efforts in the study of these astrophysical processes from the microscopic point of view (e.g., Mori \& Burkert 2000; Quilis 2004).

We present in Appendix A a few tests of the compatibility of the results with our macrospcopic approach based on integrated equations and the detailed description we can obtain from codes available in the literature that solve the microphysics of the instability processes. Hereafter, we show the utility of our technique in a real astrophysical context, because the ultimate achievement of this technique is indeed to understand the relation between the dissipative phenomena and their connection with observable quantities, the orbits of dwarf galaxies, and their CMD.

The most natural laboratory to search for real observational data is surely the LG of galaxies. The LG of galaxies are of paramount importance because of their proximity and the wealth of data available for studies of the past SFH (e.g., Grebel 1997; Orban et al. 2008). In the recent past, the acquisition of large samples of spectroscopic data made it possible to measure the internal kinematics of some of these dwarf galaxies, as well as, in a number of cases, their stellar metallicities and abundances (e.g., Koch et al. 2008; Bono et al. 2010; Koch et al. 2007) thus initiating a new generation of full chemo-dynamical models able to take both orbital parameters deduced from proper motions and internal kinematics into account. There is a rich literature for instance on determining the orbits of Magellanic Clouds after the proper motions determination by Kallivayalil et al. (2006). Pasetto et al. (2011) presented a study of the Carina dwarf spheroidal galaxy in which, taking most of the available 




Age
0
-1
-2
-3
-4
-5
-6
-7
-8
-9
-10

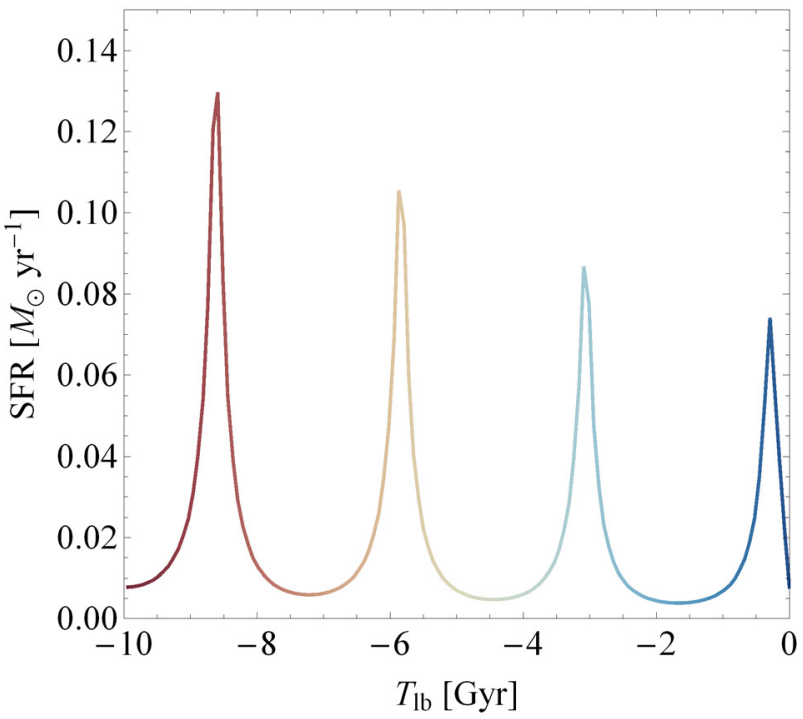

Fig. 6. The same as in Fig. 5 but for the eccentricity $e=0.75$.

observational constraints into account, we recovered both the past SFH and the internal kinematics of this dwarf galaxy in a self-consistent fashion.

With the results of Pasetto et al. (2011) in the light of the new technique we have developed here, we have recovered, for the first time self consistently the star formation history of the Carina dwarf galaxy, as a consequence of the pericentre passages that trigger SF. The technique that we adopted is based on a chemodynamical code (e.g., Berczik et al. 2003) with new star formation recipes described in detail in Pasetto et al. (2010). Our simulations reach a mass resolution of $118 M_{\odot}$, resolving the core of the dwarf galaxy with about 127000 particles (stars) over about 700000 total particles. In this approach the bursts of star formation in the evolution of Carina are the result of microscopic physical effects. The orbits of particles are integrated and some recipes rule the star formation and feedback that each particle experiences or exerts on the others. We show here that our earlier solution for the star formation history of Carina is not unique, and it can be easily achieved by our new methodology. We proceed with a few simpler assumptions than in the work of Pasetto et al. (2011), because this exercise is meant to be a qualitative


example and not a quantitative study of the importance of the relative dissipative effects (for a forthcoming work, Pasetto et al. 2012 , in prep.). We do not span the entire range of orbital parameters as in Pasetto et al. (2011); moreover, the starting model adopted for the primordial dwarf galaxy is inspired by the results of the later work, except that for the sake of simplicity we neglect here the temporal evolution of the model for the MW. The MW potential is described by the following gravitational potential

$$
\begin{aligned}
& \Phi_{\text {halo }}^{\mathrm{MW}}(R, z)=k_{1}^{2} \log \left(R^{2}+k_{2}^{2}+\frac{z^{2}}{k_{3}^{2}}\right) \\
& \Phi_{\text {disk }}^{\mathrm{MW}}(R, z)=-\frac{G M_{\text {disk }}}{\sqrt{R^{2}+\left(k_{4}+\sqrt{z^{2}+k_{5}^{2}}\right)}} \\
& \Phi_{\text {bulge }}^{\mathrm{MW}}(R, z)=-\frac{G M_{\text {bulge }}}{\sqrt{R^{2}+z^{2}}+k_{6}}
\end{aligned}
$$

with $G$ the gravitational constant and the family of parameters $\left\{k_{i}\right\}, i=2, . ., 6$, given by $\{12,0.8,6.5,0.26,0.7\}$ in $\mathrm{kpc}$, and $k_{1}=130.8$ in $\mathrm{km} \mathrm{s}^{-1}, M_{\text {bulge }}=3.4 \times 10^{10} M_{\odot}$ and 


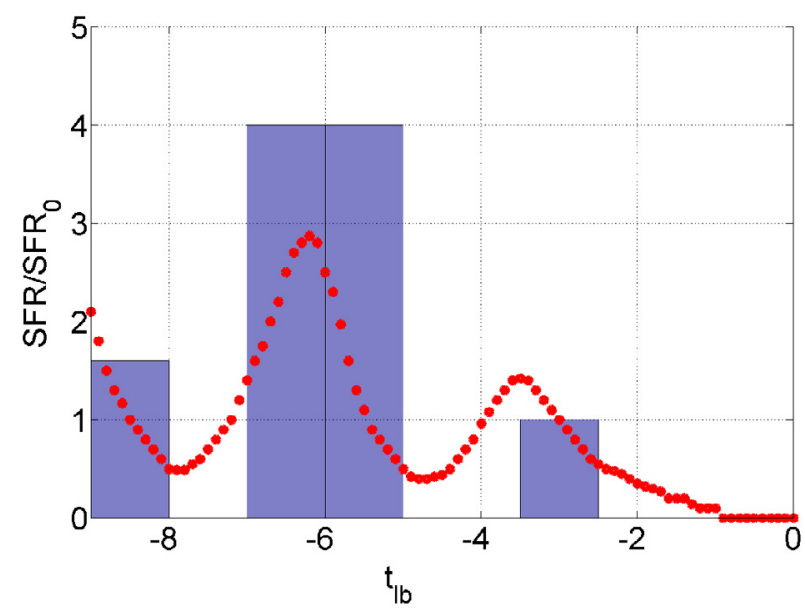

Fig. 7. Recovered episodic star formation history of the Carina dwarf galaxy superposed on the normalized histogram bars from the data of Rizzi et al. (2003) (see also Pasetto et al. 2011).

$M_{\text {disk }}=1.0 \times 10^{10} M_{\odot}$. Over this external field, only the family of orbits in the neighbourhood of the phase-space point $\mathbf{x}=\{22,89,-34\} \mathrm{kpc}, \mathbf{v}=\{-136,13,-45\}$ in a reference frame $S_{0}$ collinear with the MW potential are investigated and extracted from the family of proper motions in Table 1 of Metz et al. (2008) with the minimization action technique developed in Pasetto et al. (2011). We adopt an initial potential-density pair for Carina from the sub-family $(1,4, \gamma)$ of the Zhao-models (Zhao 1996), widely known also as the $\gamma$-models. We assume $M(r)=M_{\mathrm{bar}}\left(\frac{r}{r+r_{\mathrm{s}}}\right)^{3-\gamma}$ with $M_{\mathrm{bar}}=4.8 \times 10^{7} M_{\odot}$ the baryonic mass of the dwarf galaxy, $r_{\mathrm{s}}=0.7 \mathrm{kpc}$ and $\gamma=3 / 2$. Finally, we adopt an initial SFR $\psi_{0}=0.029 M_{\odot} \mathrm{yr}^{-1}$ at $t_{\mathrm{lb}}=10 \mathrm{Gyr}\left(t_{\mathrm{lb}}\right.$ stands for look-back time). The corresponding initial value of the pressure required to balance the cloud's destruction rate and the star formation rate is $P=0.01 P_{\odot}$. The results for the recovered SFH are plotted in Fig. 7. Finally, stellar models in system Eq. (4) and for the H-R diagrams in the low-mass regime (from 0.15 to $2.5 M_{\odot}$ ) come from the work of Bertelli et al. (2008), and for higher mass from Bertelli et al. (2009). The technique is based on the the earlier works of Bressan et al. (1993), Fagotto et al. (1994a,b), Girardi et al. (1996) but is updated in the opacities, rates of energy loss by plasma neutrinos, and nuclear network integration (see Bertelli et al. 2009, and references therein).

By straight comparison of our plot in Fig. 7 with Fig. 11 in Pasetto et al. (2011), the similarity is remarkable, thus confirming the original idea contained in that paper that the SFH of the Carina dwarf galaxy can be explained as results of pericentre passages. But the techniques are completely different. In our approach we do not integrate the orbits of the single particles. There are not microscopic particles but macroscopic analytical properties (total mass, density profiles, etc.). The star formation is the result of the pressure on the molecular cloud model, and the pressure depends on the orbit through the MW potential, the tidal tensor, as well as ram pressure, Kelvin-Helmholtz and Rayleigh-Taylor dissipative phenomena. The major difference is that the effect of the tidal field of the MW is fully accounted for by solving the EoM for the single dwarf galaxy particles in an $\mathrm{N}$ body simulation, while here it is only considered by means of a second-order perturbation, ultimately by the tidal tensor $\mathbf{T}$. More importance is given here to external instability processes that were not considered in Pasetto et al. (2011). We have two different techniques that match the same observational constraint, i.e. the star formation history. We point out another important difference with respect to the results obtained by Pasetto et al. (2011): while a high-resolution chemo-dynamical simulation requires about four months on a special hardware machine, the result presented here needed only about eight hours on a parallel cluster of processors. Moreover, the present technique can impose more straightforward constraints on the gas fraction adopted for modelling Carina than are impossible to reach because of particle resolution problems in a fully hydrodynamical NB-TSPH simulation. In this respect the new technique is superior to the one followed in Pasetto et al. (2011). The high speed of the present algorithm is partially due to the fully analytical formulation and partially to the high scalability of the code. We refer the interested reader to Appendix A for details on the parallelization performance of the code. Alternatively, the fully hydrodynamic code is best suited to studies of the internal kinematics that we have not taken into consideration here.

\section{Discussion, open questions, future developments, and conclusion}

We have developed a method and companion code able to quickly handle the major phenomena affecting the SFH of extended dwarf galaxies moving through an external environment and compared the results with those obtained from a fully hydrodynamical code (Pasetto et al. 2011). This formalism stands on a novel interpretation of the classical results for a flow past an extended spherical object (e.g., Batchelor 2000; Landau \& Lifshitz 1959), where we considered the problem in a non-inertial reference frame immersed in an external field and exploited the tidal approximation for the external gravitational interaction (e.g., Pasetto \& Chiosi 2009).

The advantage of this method, if compared to fully hydrodynamic codes, is its high speed (without imposing the restriction of a point-mass approximation), which is possible thanks to a new direction-dependent formulation for the pressure effects described in a non-inertial reference system, and that its analytical formulation offers a valuable alternative to the classical works of Gunn \& Gott (1972) or Nulsen (1982).

The technique is very suitable for imposing constraints on the orbits based on the SFH of dwarf galaxies. We have shown a possible application to the case of Carina to confirm the validity of the approach adopted. Another natural application is to study the triplet Large Magellanic Cloud, Small Magellanic Cloud, and MW by taking their SFH into account. A further possible application is to constrain the phase-space parameters of the external group of dwarf galaxies. The apparent lack of intermediate age populations in some early dwarfs around Cen A (see Crnojević et al. 2011) or in other objects for which, due to their distance, only the most luminous part of the CMD can be resolved into stars could be investigated using the structural and dynamical constraints we have proposed here. In studies of such more distant dwarfs, spectroscopic measurements of internal kinematics are still difficult to obtain and often only photometric data are available. As a consequence, investigating these important issues with complete chemo-dynamical codes is timeconsuming, whereas a fast and flexible technique and companion codes offer better chances.

Finally, while the competition of the different dissipative phenomena to produce an observable quantity is surely a target of this project, it is not the primary goal. A dwarf galaxy is affected in its orbital evolution by several phenomena that all act jointly to produce an observational result, making it difficult 
to constrain the contribution of one individual mechanism. Our technique is, by itself, not able to disentangle the relevance of all those different phenomena because, as just seen in the exercise for the Carina dwarf galaxy, the same result can be achieved with different methods. Nevertheless, this approach is a step forward in including of the stellar population algorithms to add a further observational constraint to the orbit investigation.

Of course, the simplicity of the adopted approach also comes with a few technical limitations and drawbacks that should be amended. For instance, the magnetic field can play a role in the Rayleigh-Taylor instability (Chandrasekhar 1961). Moreover, the magnetic fields might also affect the star formation efficiency by changing the Jeans instability criteria because the critical mass for collapse depends on the Alfvén and the sound speeds. Even if magnetic fields play a strong role in the collapse and fragmentation of cold clouds, the generalization of Eq. (10) to include magnetic fields is not a trivial task. Moreover, if we consider that the interstellar medium in dwarf galaxies is heterogeneous and has varying density, we expect hydrostatic turbulent pressure to act in differently way in a different regions of a dwarf galaxy (Bureau \& Carignan 2002). Finally, tightly related to the studies of star formation and environmental effects is the problem of gas removal from dwarf galaxies. If ram pressure and/or gas consumption cannot explain all the current observations, a plausible alternative could be the gas ionization by UV radiation (of stellar or Galactic origin) and by SN explosions. These effects played an important role during the epoch of reionization by increasing the Jeans mass. Unfortunately the theoretical predictions for the low-mass regime of the dwarf galaxies is strongly biased by the numerical resolution. Finally, in a cosmological context, the reionization at low redshift could have played an important role in the star formation processes (Iliev et al. 2011), which however we have neglected here to a first approximation of the problem.

Considering our finding, a natural follow-up project would be to study an extended family of orbits and model parameters to investigate the competitive role of the different dissipative phenomena acting around the MW, M31, or by considering the LG tidal interactions: the processes that affect the star formation history, and those that do not. When is a deep potential well of the dwarf galaxy massive enough to suppress the effects of the various dissipative phenomena? When do tides promote or inhibit star formation through compression/stripping? As for ram pressure, Rayleigh-Taylor, and Kelvin-Helmholtz effects, when and where are they dominant in the history of MW dwarf galaxies? These and many other questions can be investigated with this technique, for the first time in relation to synthetically generated colour-magnitude diagrams, in order to understand how to interpret the observed CMDs (Pasetto et al. 2012, in prep.).

Acknowledgements. We thank the anonymous referee for helpful comments. S.P. thanks D. Kawata for stimulating discussions and a careful reading of the manuscript. The simulations presented in this work were performed at the Leibnitz Rechenzentrum of Munich on the National Supercomputer HLRB II. E.K.G. was partially supported by Sonderforschungsbereich S.F.B. 881 "The Milky Way System" (subproject A2) of the German Research Foundation (DFG). S.P. thanks the High Performance Computing team of the HLRB-II for their support in the high-resolution simulation of Appendix A.1.

\section{References}

Anderson, M. E., \& Bregman, J. N. 2010, ApJ, 714, 320

Arnaud, M., \& Evrard, A. E. 1999, MNRAS, 305, 631

Batchelor, G. K. 2000, An Introduction to Fluid Dynamics (Cambridge

University Press)

Berczik, P., Hensler, G., Theis, C., \& Spurzem, R. 2003, Ap\&SS, 284, 865
Bertelli, G., Bressan, A. G., \& Chiosi, C. 1985, A\&A, 150, 33

Bertelli, G., Bressan, A., Chiosi, C., Ng, Y. K., \& Ortolani, S. 1995, A\&A, 301, 381

Bertelli, G., Girardi, L., Marigo, P., \& Nasi, E. 2008, A\&A, 484, 815

Bertelli, G., Nasi, E., Girardi, L., \& Marigo, P. 2009, A\&A, 508, 355

Bigiel, F., Leroy, A., Walter, F., et al. 2008, AJ, 136, 2846

Binney, J. J., \& Evans, N. W. 2001, MNRAS, 327, L27

Bono, G., Stetson, P. B., Walker, A. R., et al. 2010, PASP, 122, 651

Bradamante, F., Matteucci, F., \& D'Ercole, A. 1998, A\&A, 337, 338

Bressan, A., Fagotto, F., Bertelli, G., \& Chiosi, C. 1993, A\&AS, 100, 647

Brüns, C., Kerp, J., Kalberla, P. M. W., \& Mebold, U. 2000, A\&A, 357, 120

Bureau, M., \& Carignan, C. 2002, AJ, 123, 1316

Burkert, A., \& Ruiz-Lapuente, P. 1997, ApJ, 480, 297

Butcher, H., \& Oemler, Jr., A. 1978, ApJ, 219, 18

Byrd, G., \& Valtonen, M. 1990, ApJ, 350, 89

Cavaliere, A., \& Fusco-Femiano, R. 1976, A\&A, 49, 137

Chandrasekhar, S. 1961, Hydrodynamic and Hydromagnetic Stability (Courier Dover Publications)

Chiosi, C., Bertelli, G., Meylan, G., \& Ortolani, S. 1989, A\&A, 219, 167

Chorin, A. J., \& Marsden, J. E. 1979, A Mathematical Introduction to Fluid Mechnanics (New York: Springer)

Cioffi, D. F., \& Shull, J. M. 1991, ApJ, 367, 96

Cole, S., Lacey, C. G., Baugh, C. M., \& Frenk, C. S. 2000, MNRAS, 319, 168

Couch, W. J., Barger, A. J., Smail, I., Ellis, R. S., \& Sharples, R. M. 1998, ApJ, 497, 188

Crnojević, D., Rejkuba, M., Grebel, E. K., da Costa, G., \& Jerjen, H. 2011, A\&A, 530, A58

Davies, J. I. \& Phillipps, S. 1988, MNRAS, 233, 553

De Lucia, G., Kauffmann, G., \& White, S. D. M. 2004, MNRAS, 349, 1101

Dressler, A. 1980, ApJ, 236, 351

Dressler, A., Oemler, Jr., A., Couch, W. J., et al. 1997, ApJ, 490, 577

Einasto, J., Saar, E., Kaasik, A., \& Chernin, A. D. 1974, Nature, 252, 111

Elmegreen, B. G. 1989, ApJ, 338, 178

Elmegreen, B. G., \& Efremov, Y. N. 1997, ApJ, 480, 235

Elmegreen, B. G., \& Hunter, D. A. 2000, ApJ, 540, 814

Elmegreen, B. G., Elmegreen, D. M., Chandar, R., Whitmore, B., \& Regan, M. 2006, ApJ, 644, 879

Ettori, S., \& Fabian, A. C. 1999, MNRAS, 305, 834

Evrard, A. E. 1991, MNRAS, 248, 8P

Fagotto, F., Bressan, A., Bertelli, G., \& Chiosi, C. 1994a, A\&AS, 105, 29

Fagotto, F., Bressan, A., Bertelli, G., \& Chiosi, C. 1994b, A\&AS, 105, 39

Ferrara, A., \& Tolstoy, E. 2000, MNRAS, 313, 291

Fujita, Y. 1998, ApJ, 509, 587

Fujita, Y., \& Nagashima, M. 1999, ApJ, 516, 619

Fukugita, M., \& Peebles, P. J. E. 2006, ApJ, 639, 590

Gallart, C., Martínez-Delgado, D., Gómez-Flechoso, M. A., \& Mateo, M. 2001, AJ, 121, 2572

Girardi, L., Chiosi, C., Bertelli, G., \& Bressan, A. 1995, A\&A, 298, 87

Girardi, L., Bressan, A., Chiosi, C., Bertelli, G., \& Nasi, E. 1996, A\&AS, 117, 113

Gnedin, O. Y. 2003, ApJ, 582, 141

Goldstein, H., Poole, C., \& Safko, J. 2002, Classical mechanics, 3rd edn. (San Francisco: Addison-Wesley)

Goto, T., Okamura, S., Sekiguchi, M., et al. 2003, PASJ, 55, 757

Grcevich, J., \& Putman, M. E. 2009, ApJ, 696, 385

Grebel, E. K. 1997, in Rev. Mod. Astron., ed. R. E. Schielicke, 10, 29

Grebel, E. K., Gallagher, III, J. S., \& Harbeck, D. 2003, AJ, 125, 1926

Gunn, J. E., \& Gott, III, J. R. 1972, ApJ, 176, 1

Heitsch, F., \& Putman, M. E. 2009, ApJ, 698, 1485

Henriksen, M., \& Byrd, G. 1996, ApJ, 459, 82

Iliev, I. T., Moore, B., Gottlöber, S., et al. 2011, MNRAS, 413, 2093

Kalberla, P. M. W., \& Kerp, J. 1998, A\&A, 339, 745

Kallivayalil, N., van der Marel, R. P., \& Alcock, C. 2006, ApJ, 652, 1213

Kang, X., Jing, Y. P., Mo, H. J., \& Börner, G. 2005, ApJ, 631, 21

Kaufmann, T., Mayer, L., Wadsley, J., Stadel, J., \& Moore, B. 2006, MNRAS, 370,1612

Kippenhahn, R., \& Weigert, A. 1994, Stellar Structure and Evolution (Berlin, Heidelberg, New York: Springer-Verlag)

Klessen, R. S. 2011, EAS Pub. Ser., 51, 133 (Berlin, Heidelberg, New York: Springer-Verlag)

Klimentowski, J., Łokas, E. L., Kazantzidis, S., Mayer, L., \& Mamon, G. A. 2009, MNRAS, 397, 2015

Koch, A., Grebel, E. K., Kleyna, J. T., et al. 2007, AJ, 133, 270

Koch, A., Grebel, E. K., Gilmore, G. F., et al. 2008, AJ, 135, 1580

Krumholz, M. R., \& McKee, C. F. 2005, ApJ, 630, 250

Krumholz, M. R., \& Tan, J. C. 2007, ApJ, 654, 304

Krumholz, M. R., McKee, C. F., \& Tumlinson, J. 2008, ApJ, 689, 865

Krumholz, M. R., McKee, C. F., \& Tumlinson, J. 2009, ApJ, 693, 216 
Landau, L. D., \& Lifshitz, E. M. 1959, Fluid mechanics (London, New York: Pergamon Press)

Livio, M., Regev, O., \& Shaviv, G. 1980, ApJ, 240, L83

Mac Low, M.-M., \& Ferrara, A. 1998, in Lect. Notes Phys., IAU Colloq. 166: The Local Bubble and Beyond, ed. D. Breitschwerdt, M. J. Freyberg, \& J. Truemper (Berlin: Springer Verlag), 506, 559

Maller, A. H., \& Bullock, J. S. 2004, MNRAS, 355, 694

Maloney, P. R., \& Bland-Hawthorn, J. 1999, ApJ, 522, L81

Mayer, L. 2010, Adv. Astron., 2010

Mayer, L., Governato, F., Colpi, M., et al. 2001, ApJ, 559, 754

McKee, C. F., \& Krumholz, M. R. 2010, ApJ, 709, 308

Metz, M., Kroupa, P., \& Libeskind, N. I. 2008, ApJ, 680, 287

Mohr, J. J., Mathiesen, B., \& Evrard, A. E. 1999, ApJ, 517, 627

Moore, B., \& Davis, M. 1994, MNRAS, 270, 209

Moore, B., Katz, N., Lake, G., Dressler, A., \& Oemler, A. 1996, Nature, 379, 613

Moore, B., Lake, G., \& Katz, N. 1998, ApJ, 495, 139

Moore, B., Lake, G., Quinn, T., \& Stadel, J. 1999, MNRAS, 304, 465

Mori, M., \& Burkert, A. 2000, ApJ, 538, 559

Murali, C. 2000, ApJ, 529, L81

Murray, S. D., White, S. D. M., Blondin, J. M., \& Lin, D. N. C. 1993, ApJ, 407, 588

Nagashima, M., \& Yoshii, Y. 2004, ApJ, 610, 23

Navarro, J. F., \& Steinmetz, M. 2000a, ApJ, 538, 477

Navarro, J. F., \& Steinmetz, M. 2000b, ApJ, 528, 607

Ng, Y. K., Brogt, E., Chiosi, C., \& Bertelli, G. 2002, A\&A, 392, 1129

Nichols, M., Lin, D., \& Bland-Hawthorn, J. 2012, ApJ, 748, 149

Nidever, D. L., Majewski, S. R., Butler Burton, W., \& Nigra, L. 2010, ApJ, 723, 1618

Nulsen, P. E. J. 1982, MNRAS, 198, 1007

Oemler, Jr., A. 1974, ApJ, 194, 1

Orban, C., Gnedin, O. Y., Weisz, D. R., et al. 2008, ApJ, 686, 1030

Pasetto, S., \& Chiosi, C. 2009, A\&A, 499, 385

Pasetto, S., Chiosi, C., \& Carraro, G. 2003, A\&A, 405, 931

Pasetto, S., Grebel, E. K., Berczik, P., Spurzem, R., \& Dehnen, W. 2010, A\&A, 514, A47
Pasetto, S., Grebel, E. K., Berczik, P., Chiosi, C., \& Spurzem, R. 2011, A\&A 525, A99

Peebles, P. J. E. 1980, The large-scale structure of the universe

Peebles, P. J. E. 1993, Principles of Physical Cosmology

Phillipps, S., Edmunds, M. G., \& Davies, J. I. 1990, MNRAS, 244, 168

Portinari, L., \& Sommer-Larsen, J. 2007, MNRAS, 375, 913

Press, W. H., Teukolsky, S. A., Vetterling, W. T., et al. 1993, The Observatory, 113,214

Putman, M. E., Bland-Hawthorn, J., Veilleux, S., et al. 2003, ApJ, 597, 948

Quilis, V. 2004, MNRAS, 352, 1426

Quilis, V., \& Moore, B. 2001, ApJ, 555, L95

Quilis, V., Moore, B., \& Bower, R. 2000, Science, 288, 1617

Rasmussen, J., \& Pedersen, K. 2001, ApJ, 559, 892

Read, J. I., Wilkinson, M. I., Evans, N. W., Gilmore, G., \& Kleyna, J. T. 2006, MNRAS, 367, 387

Richter, P., Paerels, F. B. S., \& Kaastra, J. S. 2008, Space Sci. Rev., 134, 25

Rizzi, L., Held, E. V., Bertelli, G., \& Saviane, I. 2003, ApJ, 589, L85

Roediger, E., \& Brüggen, M. 2008, MNRAS, 388, L89

Roediger, E., \& Hensler, G. 2005, A\&A, 433, 875

Roediger, E., \& Hensler, G. 2008, A\&A, 483, 121

Savage, B. D., Sembach, K. R., Wakker, B. P., et al. 2003, ApJS, 146, 125

Sembach, K. R., Wakker, B. P., Savage, B. D., et al. 2003, ApJS, 146, 165

Somerville, R. S., \& Primack, J. R. 1999, MNRAS, 310, 1087

Sommer-Larsen, J. 2006, ApJ, 644, L1

Stanimirović, S., Dickey, J. M., Krčo, M., \& Brooks, A. M. 2002, ApJ, 576, 773

Steinmetz, M., \& Navarro, J. F. 1999, ApJ, 513, 555

Surdin, V. G. 1989, Astron. Nachr., 310, 381

Tonnesen, S., Bryan, G. L., \& van Gorkom, J. H. 2007, ApJ, 671, 1434

Tumlinson, J., \& Fang, T. 2005, ApJ, 623, L97

Valluri, M. 1993, ApJ, 408, 57

van den Bergh, S. 1994, ApJ, 428, 617

White, S. D. M., \& Frenk, C. S. 1991, ApJ, 379, 52

White, S. D. M., \& Rees, M. J. 1978, MNRAS, 183, 341

Zhao, H. 1996, MNRAS, 278, 488 


\section{Appendix A: Testing the code}

We group in this section a few technical tests to control the code stability and its convergence and to compare them with other literature results.

\section{A.1. Convergence tests and code scalability}

We present here the test of the convergence of the results of integrating the system of equations proposed in Eqs. (4) and a few comments on a remarkable result we obtained with the parallelization of the code. As explained in the main text, our problem is to single out the volume occupied by the orbits (differentiable functions $\boldsymbol{x}: \mathbb{R} \rightarrow \mathbb{R}^{3}$ of the real line (i.e., the time) on the configuration space $\mathbb{Q}$ ) that minimizes a $\chi^{2}$ function-difference between an observed and a synthetic CMD (convolved with distance modulus and errors). This function is clearly surjective (i.e. "onto") so that, once the volume of the parameter space is huge and multi-dimensional, the possibility of rapidly converging to a best-fit solution becomes an important issue. The structure of the code we have developed is extremely promising thanks to the scalability of the system of Eq. (4). For its integration, the classical Runge-Kutta method with adaptive stepsize was adopted (e.g., Press et al. 1993), but the mass classes were spread over several processors. Clearly, to optimize/balance the computational load, we needed to split the equations over the rank size by minimizing the latency over the available processors: $N_{\mathrm{c}}-\operatorname{Int}\left[\frac{N_{\mathrm{c}}}{N_{\mathrm{p}}}\right] N_{\mathrm{p}}$ with $N_{\mathrm{p}}$ the number of processors and $N_{\mathrm{c}}$ the number of classes. The plot is as in the Fig. A.1.

Once the performance of the code was understood, we performed a few convergence tests on the astrophysical results. Depending on the available computational resources, say the $N_{\mathrm{p}}$, we could choose the best number of processors on which to perform our integration by looking at Fig. A.1. We had the possibility of testing the lower minimum peak to the right of Fig. A.1 (for $N_{\mathrm{p}}=6500$ ). For this high-resolution test, the system of equations of Eqs. (4) was integrated with $\alpha=\frac{1}{1000}$ as defined in Sect. 2.1. The results are presented in Fig. A. 2 where four lines are plotted, for the integration of the system of Eqs. (4) with $7,65,650$, and 6500 molecular cloud classes on an E050 orbit. The test shows how the approximation adopted in our study, for $\alpha=1 / 100$, i.e., 650 molecular classes (the blue line), gives a robust consistent star formation rate with the higher resolution simulation (the red line): red and blue lines overlap almost perfectly.

On 1 May 2011 the simulation ran on 6500 processors at SGI Altix 4700 at the National Supercomputer HLRB-II, Munich (Germany) on a dedicated queue recording a peak performance of 23Tflops/second.

\section{A.2. Tests on the integro-differential system of Eqs. (4)}

Equations (4) is an integro-differential system of equations used to deduce the star formation rate of a galaxy from the total pressure $P$. While this system is conceptually stable because it is based on the fundamental principles of stellar structure and evolution (e.g., Bertelli et al. 2008, 2009), its integration is not trivial, and the numerical approaches adopted needs to be tested. Its integration on the independent variable $t$ is the most computationally time-consuming part of the code, and it was performed with distributed-memory parallelization standard techniques (message-passing interface, MPI). A serial integration of a similar system has been already performed by Fujita (1998).

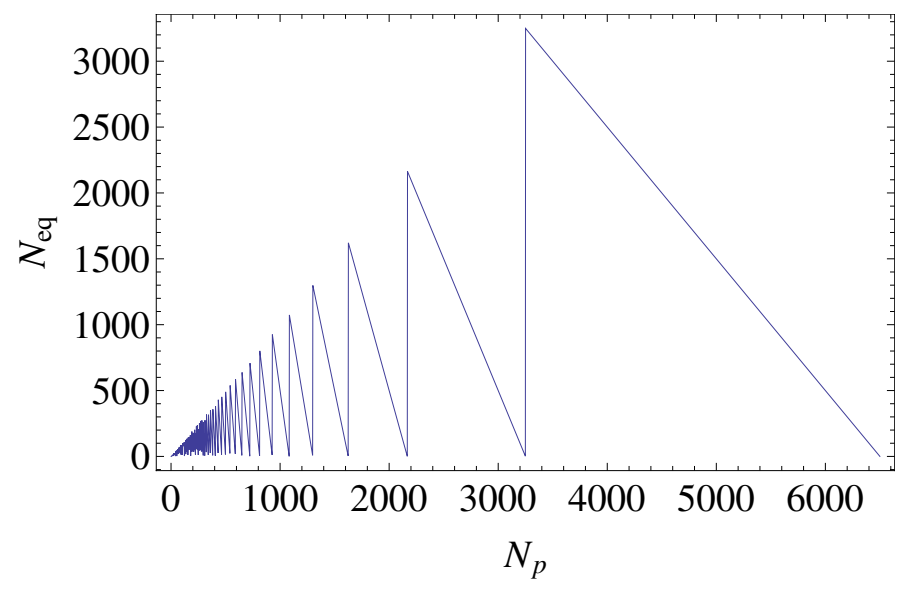

Fig. A.1. Plot of theoretical load balance between number of processors $N_{\mathrm{p}}$ and number of equations in the system (4).

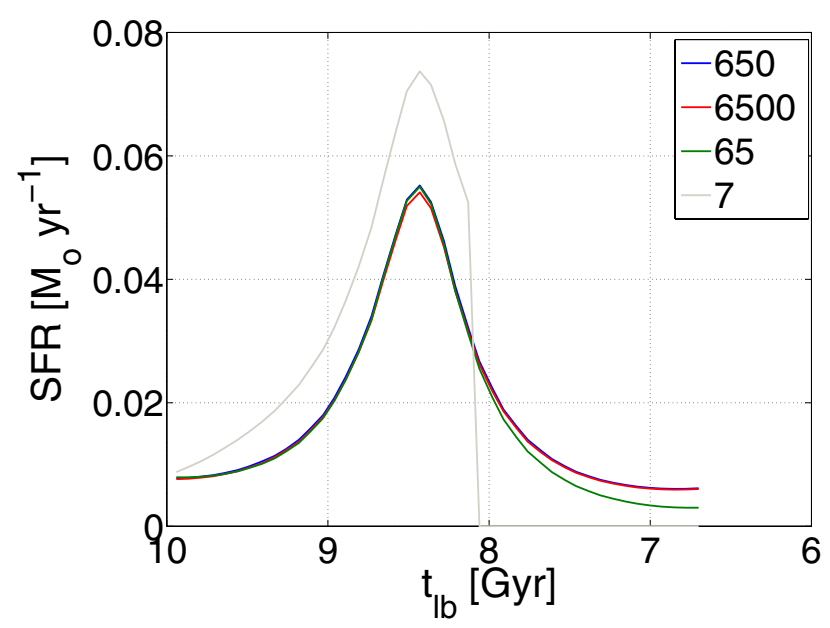

Fig. A.2. Plot of theoretical load balance between number of processors $N_{\mathrm{p}}$ and number of equations in the system (4).

Therefore, we can prepare a case test to reproduce the same result posted in Fujita (1998) by omitting the pressure determination with our Eq. (10) and instead by "injecting" into our code the synthetic pressure profile of Fig.1a (dashed line) in Fujita (1998). Thus, we eliminate the code section devoted to our original pressure determination, and only the parallel integrator of the system of Eqs. (4) is tested against the serial-independent determination originally presented in Fujita (1998). The pressure is defined piece-wise as

$P(t)=\left\{\begin{array}{cc}P_{\odot} & t \leq 0 \\ P_{\odot} e^{\frac{3}{2}(\ln 2+\ln 5) t} & 0<t \leq 2 \\ 1000 P_{\odot} & t>2 .\end{array}\right.$

We see in Fig. A.3 that our results are almost identical to the dashed line of Fig. 1b in Fujita (1998). Here the integration is performed with the Runge-Kutta method with adaptive stepsize control (e.g., Press et al. 1993) and distributed over 650 processors.

\section{A.3. Pressure from Eq. (10) and speed limits}

We assume here that the dwarf galaxy is falling in a general intra-cluster medium (ICM) or that a dwarf galaxy is passing through the MW gaseous disk. In these situations the motion 


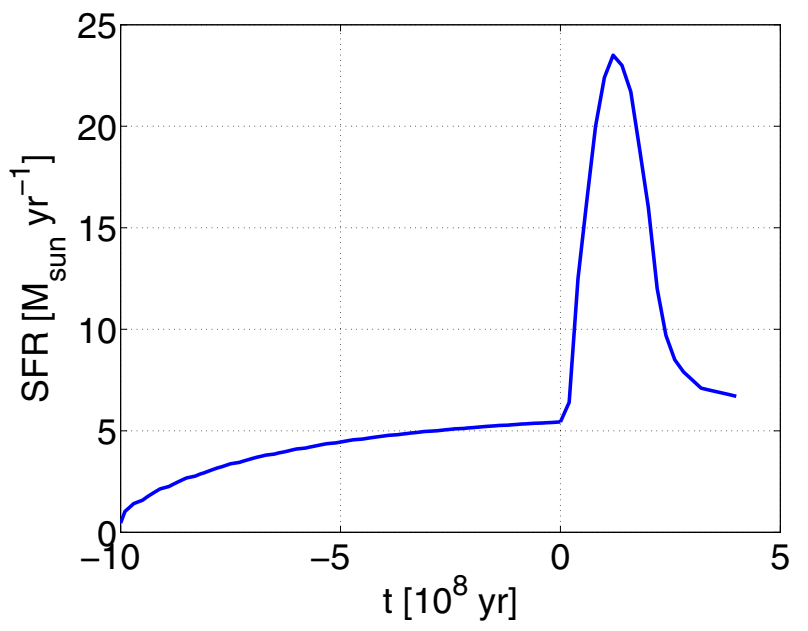

Fig. A.3. Star formation rate produced by the pressure injected from Eq. (A.1).

of the dwarf can easily be in the supersonic regime, i.e. Mach number $M_{\text {pre }}>1$, or equivalently that the flow is impacting on dwarf galaxy at supersonic velocity $v_{\mathrm{g}}>v_{\mathrm{s}, \mathrm{ICM}}$ with $v_{\mathrm{s} \text {,ICM }}$ sound speed of the flow. Moreover, we keep this exercise general by assuming a polytrophic equation of state with adiabatic index $\gamma$ for the gas flowing. The presence of a shock increases density (and pressure) by compression, while the velocity field from the supersonic becomes subsonic (we limit our arguments to normal shocks) whence Eq. (10) holds. Nevertheless, it is simple to prove that the pressure of the gas before the bow shock, $P_{\text {pre }}$, and the stagnation point $P_{\mathrm{s}}$ can be very different and Eq. (10) has to be "clothed" with supersonic formalism. We call the pressure after the surface of discontinuity (the thin shock), $P_{\text {post }}$. In this case the relations between pressures pre- and post-shock are already known (e.g., Landau \& Lifshitz 1959),

$$
\begin{aligned}
& \frac{P_{\text {post }}}{P_{\text {pre }}}=1+\frac{2 \gamma}{\gamma+1}\left(M_{\text {pre }}^{2}-1\right) \\
& M_{\text {post }}^{2}=\frac{\gamma+1+(\gamma-1)\left(M_{\mathrm{pre}}^{2}-1\right)}{\gamma+1+2 \gamma\left(M_{\mathrm{pre}}^{2}-1\right)},
\end{aligned}
$$

and we obtain the pressure between $P_{\text {post }}$ and $P_{\mathrm{s}}$ as

$$
\frac{P_{\mathrm{s}}}{P_{\text {post }}}=\left(\frac{\gamma-1}{2} M_{\text {post }}^{2}+1\right)^{\frac{\gamma}{\gamma-1}} \text {. }
$$

With $M_{\text {post }}^{2}$ and $\frac{P_{\text {post }}}{P_{\text {pre }}}$ from Eq. (A.2) we can write the relation between $\frac{P_{\mathrm{s}}}{P_{\mathrm{pre}}}$ as

$$
\begin{aligned}
\frac{P_{\mathrm{s}}}{P_{\text {pre }}} & =\frac{P_{\mathrm{s}}}{P_{\mathrm{post}}} \frac{P_{\mathrm{post}}}{P_{\mathrm{pre}}} \\
& =\left(\frac{\gamma+1}{2} M_{\mathrm{pre}}^{2}\right)^{\frac{\gamma}{\gamma-1}}\left(1+\frac{2 \gamma}{\gamma+1}\left(M_{\mathrm{pre}}^{2}-1\right)\right)^{-\frac{1}{\gamma-1}}
\end{aligned}
$$

whose plot is the same as in Fig. A.4. As is evident, the preshock pressure and the pressure at the stagnation point are not of the same order of magnitude even if the post shock velocity field is subsonic because the flow is compressed and its pressure (and density) increases passing through the shock and after the shock before reaching the stagnation point. As seen in Fig. A.4 this

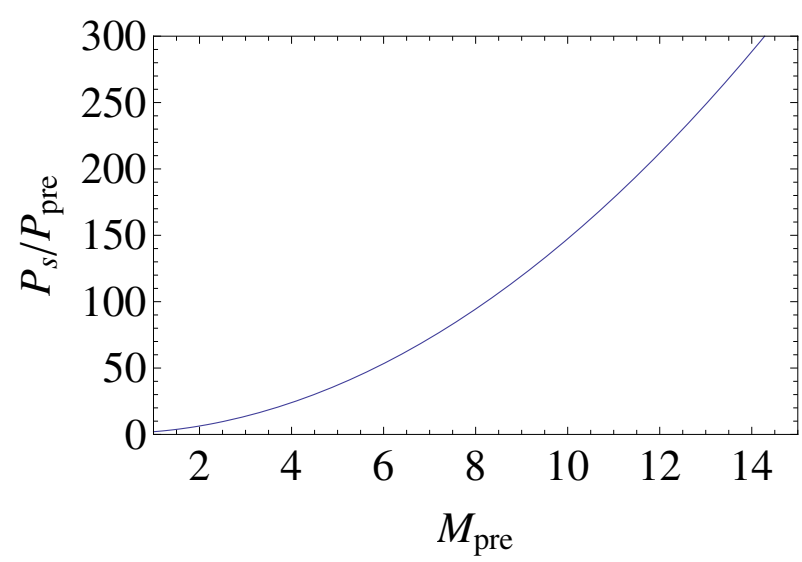

Fig. A.4. Trend between of the ratio between pressure at the stagnation point and pre-shock pressure as a function of the Mach number of the gas where the dwarf galaxy is moving.

pressure remains about the same only for subsonic or weakly supersonic regime, which represent the limit of validity of Eq. (10), before supersonic correction has to be implemented.

\section{Appendix B: Star formation efficiency and disruption time relation}

The connection between star formation processes and molecular clouds is a very fertile research topic encompassing observations (e.g., Bigiel et al. 2008), theory (e.g., Krumholz \& McKee 2005), and experimental/numerical works (e.g., Klessen 2011). In our approach we revisited a work by Elmegreen \& Efremov (1997) chosen for its simplicity. Other literature results can be similarly implemented. Let $M_{i}$ be the cloud's initial mass as defined in Sect. 2.1, for which we assumed a constant star formation rate $\varsigma_{i}$ in the integration interval $\mathrm{d} t$ (see also Elmegreen 1989; Krumholz \& McKee 2005; Krumholz \& Tan 2007). Following Surdin (1989), the erosion rate is proportional to the luminosity $L_{\mathrm{s}}$ of embedded stars with total mass $M_{\mathrm{star}, i}$ divided by the specific mass binding energy $M_{i} \sigma^{2}$ with $\sigma$ dispersion velocity. The equation for the rate of change of gas mass in the cloud considered is

$\frac{\mathrm{d} M_{i}}{\mathrm{~d} t}=-\varsigma_{i}-A_{i} \frac{L_{i}}{\sigma^{2}}$

and the luminosity is

$L_{i}(t)=\int_{0}^{t} \varsigma_{i} \Lambda_{i}\left(t-t^{\prime}\right) \mathrm{d} t^{\prime}$

where $\Lambda_{i}(t)$ is the luminosity-to-mass ratio of a population of stars generated by the cloud class at the instant $t$ that we obtained as explained in Sect. 3. We drop for the moment the subscript $i$ to simplify the notation. From the previous Eqs. (B.1) and (B.2) we get

$\frac{\mathrm{d} M}{\mathrm{~d} t}=-\varsigma\left(1+\frac{A}{\sigma^{2}} \int_{0}^{t} \Lambda\left(t-t^{\prime}\right) \mathrm{d} t^{\prime}\right)$

which can be integrated to give

$M(t)=M(0)-\varsigma\left(t+\frac{A}{\sigma^{2}} \int_{0}^{t} \mathrm{~d} t^{\prime} \int_{0}^{t^{\prime}} \Lambda\left(t^{\prime}-t^{\prime \prime}\right) \mathrm{d} t^{\prime \prime}\right)$ 
where the double integral is more easily numerically com- and puted as

$$
\int_{0}^{t} \mathrm{~d} t^{\prime} \int_{0}^{t^{\prime}} \Lambda\left(t^{\prime}-t^{\prime \prime}\right) \mathrm{d} t^{\prime \prime}=t \int_{0}^{t} \Lambda\left(t-t^{\prime}\right) \mathrm{d} t^{\prime}-\int_{0}^{t} t^{\prime} \Lambda\left(t^{\prime}\right) \mathrm{d} t^{\prime}
$$

$\alpha \equiv \frac{t_{0} \varsigma}{M(0)}$

$\beta \equiv \frac{A \Lambda_{0}}{\sigma^{2}} \frac{M(0)}{\varsigma}$

to give

$$
\begin{aligned}
M(t)= & M(0)-\varsigma\left(t+\frac{A}{\sigma^{2}}\left(t \int_{0}^{t} \Lambda\left(t-t^{\prime}\right) \mathrm{d} t^{\prime}\right.\right. \\
& \left.\left.-\int_{0}^{t} t^{\prime} \Lambda\left(t^{\prime}\right) \mathrm{d} t^{\prime}\right)\right)
\end{aligned}
$$

for every $i$. We can also find a quicker approach based on an interpolation function in the work of Girardi et al. (1995, their Fig. 13) as recently in Elmegreen et al. (2006), which we can also adopt when necessary to speed up the code. By using a power law like $\Lambda(t)=\Lambda_{0}\left(\frac{t}{t_{0}}\right)^{-\lambda}, \lambda \in[0,1[$, the integrals in Eq. (B.6) can be carried out analytically. We observe that the destruction time $\tau$ of the molecular clouds can be defined in an implicit way as the instant $\tilde{t} \mid M(\tilde{t})=0$

$$
\begin{aligned}
& -\tilde{t} \varsigma-\frac{A \tilde{t}^{2} \Lambda_{0} \varsigma}{(\lambda-1)(\lambda-2) \sigma^{2}}\left(\frac{\tilde{t}}{t_{0}}\right)^{-\lambda}+M(0)=0 \\
& \Leftrightarrow 1-\alpha \tau-\beta \frac{\alpha^{2}}{(\lambda-1)(\lambda-2)} \tau^{2-\lambda}=0,
\end{aligned}
$$

where we exploited the following definitions:

with $t_{0} \sim 10^{7} \mathrm{yr}$ as a fixed parameter from Girardi et al. (1995) and $\lambda \sim 0.6$ for $t>t_{0}$ and $\lambda=0$ for $t<t_{0}$ where the solution is analytical (a quadratic equation). Apart from the difference in the mathematical formalism laid out here, the contents then follow exactly as in Elmegreen \& Efremov (1997) to which we refer the reader for an extended discussion. We point out here that we can define the efficiency as

$\varepsilon \equiv \frac{\varsigma}{M(0)} t$

The two functions defined in (B.8) are of interest due to their dependence on pressure impacting the orbital mass of the dwarf galaxies in our LG. We assume the following functional dependence (Elmegreen 1989; Elmegreen \& Efremov 1997):

$\alpha=\alpha(M, P)=\alpha_{0}\left(\frac{M}{10^{5} M_{\odot}}\right)^{-1 / 4}\left(\frac{P}{P_{\odot}}\right)^{3 / 8}$
$\beta=\beta(M, P)=\beta_{0}\left(\frac{M}{10^{5} M_{\odot}}\right)^{-1 / 4}\left(\frac{P}{P_{\odot}}\right)^{-5 / 8}$,

with dimensionless constants $\alpha_{0}=0.1, \beta_{0}=180, P_{\odot}=3 \times$ $10^{4} k_{\mathrm{B}} \mathrm{cm}^{-3} \mathrm{~K}$, and $k_{\mathrm{B}}$ as the Boltzmann constant.

$\tau \equiv \frac{t}{t_{0}}$, 